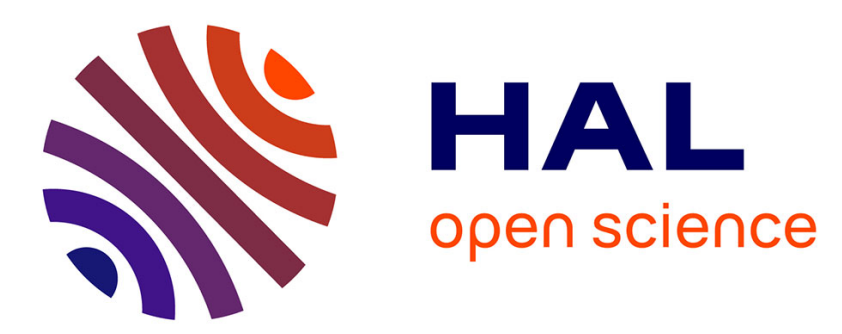

\title{
Effects of radiative heat transfer on the structure of turbulent supersonic channel flow
}

S. Ghosh, R. Friedrich, M. Pfitzner, Chr Stemmer, B. Cuenot, Mouna El-Hafi

\section{To cite this version:}

S. Ghosh, R. Friedrich, M. Pfitzner, Chr Stemmer, B. Cuenot, et al.. Effects of radiative heat transfer on the structure of turbulent supersonic channel flow. Journal of Fluid Mechanics, 2011, 677, pp.417444. 10.1017/jfm.2011.92. hal-01712147

\section{HAL Id: hal-01712147 \\ https://hal.science/hal-01712147}

Submitted on 8 Nov 2019

HAL is a multi-disciplinary open access archive for the deposit and dissemination of scientific research documents, whether they are published or not. The documents may come from teaching and research institutions in France or abroad, or from public or private research centers.
L'archive ouverte pluridisciplinaire HAL, est destinée au dépôt et à la diffusion de documents scientifiques de niveau recherche, publiés ou non, émanant des établissements d'enseignement et de recherche français ou étrangers, des laboratoires publics ou privés. 


\title{
Effects of radiative heat transfer on the structure of turbulent supersonic channel flow
}

\author{
S. GHOSH ${ }^{1}$, R. FRIEDRICH ${ }^{1} \dagger$, M. PFITZNER ${ }^{2}$, \\ CHR. STEMMER ${ }^{1}$, B. CUENOT ${ }^{3}$ AND M. EL HAFI ${ }^{4}$ \\ ${ }^{1}$ Lehrstuhl für Aerodynamik, TU München, Boltzmannstr 15, 85748 Garching, Germany \\ ${ }^{2}$ Institut für Thermodynamik, Universität der Bundeswehr München \\ Werner-Heisenberg-Weg 39, 85577 Neubiberg, Germany \\ ${ }^{3}$ CERFACS, 42 Avenue G. Coriolis, 31057 Toulouse, France \\ ${ }^{4}$ Laboratoire de Génie des Procédés des Solides Divisés, Ecole des Mines d'Albi Carmaux, \\ 81013 Albi, France
}

The interaction between turbulence in a minimal supersonic channel and radiative heat transfer is studied using large-eddy simulation. The working fluid is pure water vapour with temperature-dependent specific heats and molecular transport coefficients. Its line spectra properties are represented with a statistical narrow-band correlated-k model. A grey gas model is also tested. The parallel no-slip channel walls are treated as black surfaces concerning thermal radiation and are kept at a constant temperature of $1000 \mathrm{~K}$. Simulations have been performed for different optical thicknesses (based on the Planck mean absorption coefficient) and different Mach numbers. Results for the mean flow variables, Reynolds stresses and certain terms of their transport equations indicate that thermal radiation effects counteract compressibility (Mach number) effects. An analysis of the total energy balance reveals the importance of radiative heat transfer, compared to the turbulent and mean molecular heat transport.

Key words: compressible turbulence, large-eddy simulation

\section{Introduction}

In turbulent gaseous combustion systems, radiative heat transfer often plays an important role besides heat transfer by conduction and convection. While heat conduction is a short-range phenomenon, accomplished by molecules which on average travel the distance of a mean free path $\left(O\left(10^{-10} \mathrm{~m}\right)\right.$ under standard conditions) before they exchange energy in a collision, turbulent heat transport by convection is managed by eddies of macroscopic size, which cover distances ranging from the Kolmogorov microscale to a turbulent integral scale. Thermal radiation is a phenomenon of much longer range than the other two, in general, because photons can travel long distances before they interact with molecules. As a consequence, partial differential equations (the compressible Navier-Stokes equations) suffice to describe heat conduction and convection mechanisms, while an integro-differential equation is needed to predict the directional dependence of the radiative intensity inside absorbing/emitting gases. A further complication arises from the fact that radiative properties of gases vary strongly with the frequency or wavenumber of the 
radiation (Modest 2003). Due to these difficulties which imply enormous costs of numerical simulations, effects of radiative heat transfer are frequently predicted by strongly simplified models or are neglected even in situations where they play a role. There are indeed many examples in engineering science and technical applications where thermal radiation cannot be neglected, e.g. in combustion systems such as furnaces, boilers, scramjets and rocket engines. Coelho (2007) provides an extensive overview of the current understanding of the turbulence-radiation interaction (TRI) and its modelling in reactive low-Mach-number flows. Among the many interesting issues discussed in this overview, two aspects of TRI may be quoted here, namely the influence of heat radiation on the flow and species concentration fields on one hand and the effect of the fluctuating species and temperature fields on radiation on the other. While most work on TRI has been devoted to the influence of turbulence on radiation, little work has concentrated on the question of how radiation affects turbulence variables. It is the aim of this work to focus especially on the behaviour of the turbulence structure under the influence of thermal radiation in inert, compressible, wall-bounded shear layers.

The most accurate prediction technique for TRI is undoubtedly the direct numerical simulation (DNS) of a turbulent flow, the photon Monte Carlo method for radiative transfer ( $\mathrm{Wu}$ et al. 2005) and spectral radiation gas properties based on highresolution measurements. Such an accurate approach is not feasible at present due to the prohibitive numerical requirements, even for simple flow configurations where turbulence is inhomogeneous in one space direction only. A good compromise with respect to accuracy and cost is achieved by performing a large-eddy simulation (LES) of the flow field, applying the method of discrete ordinates (DOM) (Modest 2003; Coelho 2007) to compute the radiative transfer equation (RTE) for an absorbing/emitting gas and the statistical narrow-band correlated-k (SNB-cK) model to account for the radiative properties of the gas. This approach has been mostly adopted here, except for an attempt to reach higher optical thickness by using a simple grey gas model with an artificially increased absorption coefficient.

The paper is organized as follows: the governing equations describing compressible turbulent flow of a single-component gas that emits/absorbs thermal radiation are presented in $\S 2(\S 2.1)$, together with the LES approach for the flow field $(\S 2.2)$. Numerical and computational details in $\S 3$ comprise a description of the NavierStokes and RTE solvers (\$3.1) and of the flow configuration ( $\$ 3.2)$. Section 4 is devoted to results for mean and instantaneous quantities and starts with transport equations for mean momentum, energy and Reynolds stresses $(\S 4.1)$. Results for low and high supersonic channel flow of water vapour with and without radiation are presented in $\$ \S 4.2$ and 4.3. Snapshots of emitted and absorbed radiative energies between the channel walls are contrasted with the instantaneous temperature fields in $\S 4.4$. Section 4.5 discusses the effect of an artificially increased Planck mean absorption coefficient on certain aspects of the mean momentum and heat transport. Conclusions are drawn in $\S 5$.

\section{Mathematical model}

\subsection{Governing equations}

In Cartesian tensor notation, the compressible Navier-Stokes equations for a singlecomponent thermally radiating gas read

$$
\frac{\partial \rho}{\partial t}+\frac{\partial \rho u_{j}}{\partial x_{j}}=0
$$




$$
\begin{gathered}
\frac{\partial \rho u_{i}}{\partial t}+\frac{\partial \rho u_{i} u_{j}}{\partial x_{j}}-\frac{\partial}{\partial x_{j}}\left(-p \delta_{i j}+\tau_{i j}\right)=f_{i}, \\
\frac{\partial \rho E}{\partial t}+\frac{\partial \rho E u_{j}}{\partial x_{j}}-\frac{\partial}{\partial x_{j}}\left(-u_{i} p \delta_{i j}+u_{i} \tau_{i j}-q_{j}\right)=u_{i} f_{i} .
\end{gathered}
$$

The set (2.1)-(2.3) of coupled differential equations describes the transport of mass, momentum and total energy $\left(\rho E=\rho\left(e+0.5 u_{i} u_{i}\right)\right)$. It is supplemented by Newton's viscous stress tensor $\left(\tau_{i j}\right)$, the sum $\left(q_{j}=q_{j, C}+q_{j, R}\right)$ of heat flux vectors for conduction and radiation and the caloric and thermal equations of state:

$$
\begin{gathered}
\tau_{i j}=\mu\left(\frac{\partial u_{i}}{\partial x_{j}}+\frac{\partial u_{j}}{\partial x_{i}}\right)+\left(\kappa-\frac{2}{3} \mu\right) \frac{\partial u_{k}}{\partial x_{k}} \delta_{i j}, \\
q_{j}=-\lambda \frac{\partial T}{\partial x_{j}}+q_{j, R}, \\
\mathrm{~d} e=c_{v} \mathrm{~d} T, \quad p=\rho R T .
\end{gathered}
$$

In (2.1)-(2.6), $\rho, u_{i}, p, T$ and $e$ denote density, velocity, pressure, temperature and internal energy, respectively. $R$ is the gas constant per unit mass. Dynamic and bulk viscosities, $\mu, \kappa$, thermal conductivity, $\lambda$, and specific heat at constant volume, $c_{v}$, depend on temperature. While the molecular transport coefficients are computed efficiently using the code EGLib (Ern \& Giovangigli 1995), polynomial expressions are used to specify the temperature dependence of specific heats (Gardiner 1984). $f_{i}$ represents a body force which is needed to drive a fully developed channel flow and is specified in $\S 3.2$. The radiative source term, $\partial q_{j, R} / \partial x_{j}$, in the energy equation is obtained by integrating the RTE over all wavenumbers and directions in which radiation propagates. For an emitting-absorbing and non-scattering gas, the RTE reads (Modest 2003)

$$
\frac{\mathrm{d} I_{\eta}}{\mathrm{d} s}=\kappa_{\eta} I_{b \eta}-\kappa_{\eta} I_{\eta}
$$

The spectral radiation intensity, $I_{\eta}$, depends on the wavenumber, $\eta$, and on the direction $s$ in which radiation propagates. Its time-dependency is neglected because the speed of light is much larger than any flow velocity. $\kappa_{\eta}$ and $I_{b, \eta}$ denote the spectral absorption coefficient and Planck's black-body radiation intensity, respectively. The first term on the right-hand side of (2.7) describes the gain of radiation intensity via emission and the second term describes the loss by absorption. The radiative source term is obtained from (2.7) through integration over wavenumber $\eta$ and solid angle $\Omega$,

$$
\operatorname{div} \boldsymbol{q}_{R}=\int_{0}^{\infty} \kappa_{\eta}\left(4 \pi I_{b \eta}-\int_{4 \pi} I_{\eta} \mathrm{d} \Omega\right) \mathrm{d} \eta=\underbrace{4 \kappa_{P} \sigma T^{4}}_{\text {emission }}-\underbrace{\int_{0}^{\infty} \int_{4 \pi} \kappa_{\eta} I_{\eta} \mathrm{d} \Omega \mathrm{d} \eta}_{\text {absorption }},
$$

where $\kappa_{P}$ defines Planck's absorption coefficient which is an average over all wavenumbers:

$$
\kappa_{P}=\frac{\int_{0}^{\infty} \kappa_{\eta} I_{b \eta} \mathrm{d} \eta}{\int_{0}^{\infty} I_{b \eta} \mathrm{d} \eta}=\frac{\pi}{\sigma T^{4}} \int_{0}^{\infty} \kappa_{\eta} I_{b \eta} \mathrm{d} \eta .
$$

$\sigma$ is the Stefan-Boltzmann constant. 


\subsection{LES approach}

LES of a compressible turbulent flow needs modelling of many unclosed terms. Techniques which circumvent the modelling of each individual unknown term in the governing LES equations are therefore of great value. They may be of a purely mathematical nature and need not make use of the flow physics in modelling effects of the unresolved scales. The approximate deconvolution method presented by Stolz \& Adams (1999) is such a technique. We use a variant of this method here, namely the explicit filtering technique of Mathew et al. (2003) and briefly discuss its main characteristics using a generic one-dimensional transport equation for a variable $u(x, t)$ as an example:

$$
\frac{\partial u}{\partial t}+\frac{\partial f(u)}{\partial x}=0
$$

where $f(u)$ is a nonlinear function. Computing a low-wavenumber solution, as in LES, implies a spatial filtering of (2.10), to get

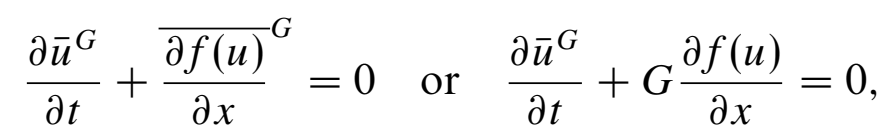

with a low-pass filter kernel $G\left(x-x^{\prime}, \Delta\right)$, its filter width $\Delta$ and the filtered (or LES) variable,

$$
\bar{u}^{G}=G * u=\int G\left(x-x^{\prime}, \Delta\right) u\left(x^{\prime}\right) \mathrm{d} x^{\prime} .
$$

Stolz \& Adams (1999) close (2.11) by using a deconvolved field $u^{*}$ which approximates $u$ in the nonlinear term of (2.11) and is given by

$$
u \simeq u^{*}, \quad u^{*}(x, t)=Q_{N} * \bar{u}^{G}=Q_{N} * G * u, \quad Q_{N}=\sum_{\nu=0}^{N}(I-G)^{v} \approx G^{-1} .
$$

$G$ is a second-order Pade filter containing a parameter $\alpha$ and $Q_{N}$ is the approximate inverse of $G$, obtained from a van Cittert series and truncated at $N=5$ or 6 .

In subsequent publications (Adams \& Leonard 1999; Stolz, Adams \& Kleiser 2001), the authors add a relaxation term on the right-hand side of (2.11) to provide the proper transfer of energy at the cutoff wavenumber between large and small scales and to prevent divergence of the solution (regularization). In Mathew et al. (2003) no relaxation term is needed and the fact that the desired dissipative action can also be achieved by filtering the numerical solution explicitly at every time step with a composite filter, $\left(Q_{N} * G\right)^{2}$, to get the LES variable $\bar{u}^{Q G}=\left(Q_{N} * G\right)^{2} * u$ is used. As seen in figure $1,\left(Q_{N} * G\right)^{2}$ acts as a useful low-pass filter, i.e. it is flat over a significant part of the represented wavenumber range and then falls off smoothly at the high wavenumber end. This filter also has the advantage that the LES result tends uniformly to the DNS result as the grid is refined and the filter cutoff is moved towards the highest wavenumbers. It is, however, crucial that a high-resolution numerical scheme can be used for spatial discretization so that the scheme does not filter out any low wavenumber content. In the present computations the Fourier transform of $G$ has the following form:

$$
\hat{G}(k)=\left(\alpha+\frac{1}{2}\right) \frac{1+\cos k}{1+2 \alpha \cos k}, \quad \alpha=0.2,
$$

in periodic directions. In the non-periodic direction, $G$ has a form proposed by Stolz (2000). 


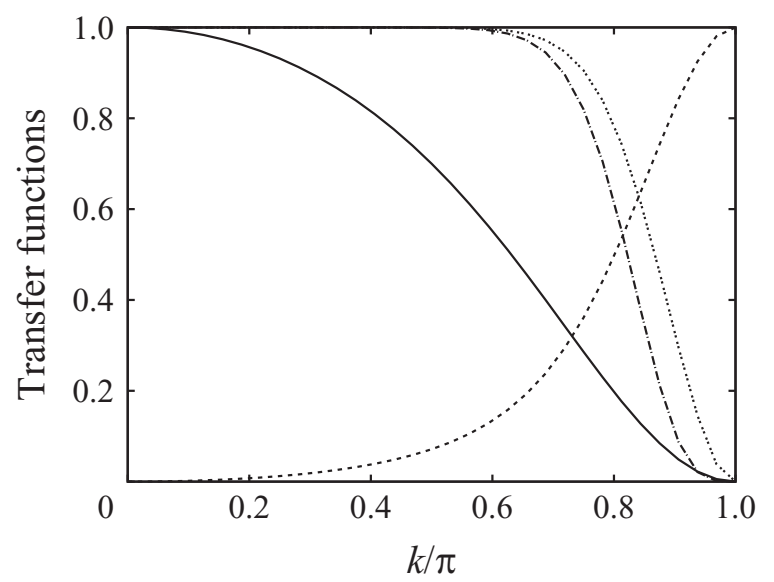

FIGURE 1. Transfer functions of filters and their approximate inverse in periodic direction. $\alpha=0.2$ and $N=6:-, G ;-.-, Q_{N} ; . . . ., Q_{N} * G ; \cdot . \cdot-,,\left(Q_{N} * G\right)^{2}$.

Following Coelho (2009), low-pass filtering of the radiation transfer equation (2.7) leads to subgrid-scale contributions for absorption and emission of the form (where the overbar here denotes a suitable low-pass filtering operation),

$$
\left(\overline{\kappa_{\eta} I_{\eta}}-\overline{\kappa_{\eta}} \overline{I_{\eta}}\right), \quad\left(\overline{\kappa_{\eta} I_{b \eta}}-\overline{\kappa_{\eta}} \overline{I_{b \eta}}\right) .
$$

Coelho (2009) has discussed several closures for these terms and has concluded that an optically thin fluctuation approximation in the LES context (which neglects the subgrid-scale contributions to the absorption term) may yield excellent predictions. He also pointed out that errors arising from the complete neglect of TRI in the filtered RTE are far less important than those resulting from ignoring TRI in the statistically averaged RTE in Reynolds averaged Navier-Stokes (RANS) calculations. In view of this fact we do not model any of the subgrid-scale contributions here and solve the RTE using the described explicitly filtered variables.

\section{Numerical and computational details}

\subsection{Navier-Stokes and RTE solvers}

The compressible Navier-Stokes equations (2.1)-(2.6) are solved numerically in a characteristics-based form proposed by Sesterhenn (2001). The compact sixth-order central scheme of Lele (1992) is chosen to discretize the convection and molecular transport terms, respectively. A third-order low-storage Runge-Kutta scheme of Williamson (1980) advances the solution in time. At each time step the discrete values of $p, T$ and $u_{i}$ defined on the Cartesian LES grid are filtered using the composite filter $\left(Q_{N} * G\right)^{2}$, so that the primitive LES-variables, $\bar{p}^{Q G}, \bar{T}^{Q G}$ and $\bar{u}_{i}{ }^{Q G}$, are obtained.

We solve the RTE as in (2.7), using the DOM, implemented in the code PRISSMA and the filtered pressure and temperature, $\bar{p}^{Q G}$ and $\bar{T}^{Q G}$, respectively. The threedimensional RTE is solved on a Cartesian, structured mesh using finite-volume discretization. A second-order Diamond mean flux scheme (Joseph et al. 2005) is used for the calculation of radiative intensity along a line of sight. Spectral integration can be performed in PRISSMA using global spectral models or a SNB-cK model (Liu, Smallwood \& Gulder 2000). The latter is mostly used here for its accuracy, although it is very expensive compared to global models. The parameters for the statistical narrow-band model used here are described in Soufiani \& Taine (1997). The flow field and the radiation field are directly coupled using Message Passing Interface (MPI) such that PRISSMA gets the pressure and temperature fields at about every 
characteristic convective time interval and the compressible LES solver receives the radiative source term. This characteristic time interval is defined as $\Delta t_{c}=\Delta x / \bar{u}_{m}$, where $\Delta x$ is the streamwise grid spacing and $\bar{u}_{m}$ is the bulk velocity. The flow solver uses domain decomposition, while the radiation solver employs parallelization in wavenumbers, i.e. in narrow bands while using the SNB-cK model.

Our LES technique has been successfully tested against DNS data for supersonic turbulent channel flow in Mathew et al. (2003). It has also been applied and again successfully validated for supersonic pipe flow (Ghosh, Sesterhenn \& Friedrich 2006) and for nozzle flow (Ghosh, Sesterhenn \& Friedrich 2008). The PRISSMA code has been used and validated in Amaya et al. (2010). The numerical scheme implemented in PRISSMA is detailed and tested in Joseph et al. (2005). In Jensen et al. (2007), the DOM is compared with other methods to solve the RTE, and it is shown that DOM using high-order quadrature provides accuracy levels comparable to the quasi-exact methods (Monte Carlo and ray tracing).

We would like to point out that our notation will be simplified from here onwards, in the sense that the specification of an LES variable by an overbar $\left(\bar{f}^{Q G}\right)$ is suppressed. All variables without an overbar are henceforth low-pass filtered timeand space-dependent quantities.

\subsection{Flow configuration}

The prediction of TRI in fully developed channel flow, using time-dependent numerical simulations, has not been given much attention in the past. The only two publications known to the authors, in which DNS/LES is used, deal with incompressible turbulence, its interaction with and its modification by thermal radiation. Gupta, Modest \& Haworth (2009) use LES to investigate reacting and non-reacting turbulent channel flows at a friction Reynolds number of 186 under conditions where composition and temperature do not affect the hydrodynamics (one-way coupling). The RTE is solved using a spherical harmonics (P1) method, and radiation properties correspond to a fictitious grey gas with a composition- and temperature-dependent Planck mean absorption coefficient that mimics that of a typical hydrocarbon-air combustion product. Simulations have been performed for different optical thicknesses and it was found that in the absence of chemical reaction, radiation modifies the mean temperature profile, but has little effect on temperature fluctuations and TRI. On the other hand, chemical reaction enhances the importance of TRI via stronger composition and temperature fluctuations. Amaya et al. (2010) perform DNS of a reacting turbulent minimal channel flow with and without radiative source terms. The RTE is solved using the DOM and the radiation model used is the global spectral FS-SNBcK model (Full spectrum statistical narrow band correlated-k model, Poitou et al. 2009) which forms a compromise between cost and accuracy. Radiation and flow dynamics are directly coupled in the sense that the Navier-Stokes solver receives the radiative source term every convective characteristic time, whereas the radiation solver gets the temperature, pressure and molar concentrations of the species fields. Specific features of the incompressible flow are internal heat sources to achieve a bulk temperature of $2000 \mathrm{~K}$, a friction Reynolds number of 400 , a low optical thickness and isothermal, black walls, kept at a temperature of $1750 \mathrm{~K}$. The working gas is a mixture of seven reacting species typical of many industrial applications: $\mathrm{CO}, \mathrm{CO}_{2}, \mathrm{H}, \mathrm{H}_{2}, \mathrm{H}_{2} \mathrm{O}, \mathrm{OH}$ and $\mathrm{N}_{2}$. As a result of the low optical thickness of the configuration, no coupling is detected between the radiation term and other energy conservation contributions, so that the contribution of the radiative heat fluxes at the walls can be calculated independently. 


$\begin{array}{lccccc}\text { Flow } & R e_{\tau} & M_{\tau} & R e_{m} & M_{m} & \tau_{H} \\ \text { Channel 1 } & 1026(1041) & 0.0681(0.068) & 16477(17059) & 1.26(1.32) & 0.006 \\ \text { Channel 2 } & 1368(1398) & 0.119(0.1177) & 17451(17590) & 2.88(2.92) & 0.003\end{array}$

TABLE 1. Flow parameters for supersonic turbulent channel flow of water vapour with radiative heat transfer (values in brackets correspond to cases without radiation).

The present configuration is that of a supersonic turbulent flow in a minimal channel (Jimenez \& Moin 1991) with pure water vapour (being an important product of combustion, besides $\mathrm{CO}_{2}$ ) as working fluid and black, no-slip surfaces kept at a constant temperature of $1000 \mathrm{~K}$. To mimic a high-altitude supersonic flight situation and to keep the Reynolds number sufficiently low, the pressure level is kept at 0.05 bar. High temperatures are naturally created at supersonic speed in the channel core by kinetic energy dissipation. Since the radiation model selected is mostly the timeconsuming SNB-cK model (Liu et al. 2000), DNS is not feasible and LESs which are fully coupled with thermal radiation computations are performed instead. Two sets of friction Reynolds and Mach numbers, $R e_{\tau}$ and $M_{\tau}$, have been selected (Channels 1 and 2) to compute effects of radiation on the turbulent-flow structure by comparison with corresponding cases where thermal radiation is turned off. In table 1 we present the friction Reynolds and Mach numbers for the case with radiation and in brackets for the case without radiation. A special feature of compressible channel flow is that these parameters cannot be chosen independently, even in the case without radiation. This is obvious from their definitions:

$$
R e_{\tau}=\frac{\bar{\rho}_{w} u_{\tau} H}{\mu_{w}}, \quad M_{\tau}=\frac{u_{\tau}}{\sqrt{\gamma R T_{w}}}, \quad u_{\tau}=\sqrt{\frac{\bar{\tau}_{w}}{\bar{\rho}_{w}}} .
$$

While the dynamic viscosity and the speed of sound at wall temperature are constants, the mean density at the wall, appearing in $R e_{\tau}$ explicitly and in $M_{\tau}$ via the friction velocity, is a result of the computation and cannot be specified in advance. The wall-shear stress is related to the mean streamwise pressure gradient which drives the flow in the form of a space/time constant body force in (2.2). For fully developed channel flow, with the channel half-width $H$, we have

$$
-\frac{\partial \bar{p}}{\partial x}=\frac{\bar{\tau}_{w}}{H}=f_{i} \delta_{i x} .
$$

When this body force is used in the momentum and energy equations (2.2) and (2.3), the pressure $p$ fluctuates along $x$ around a mean value. It is this body force which is kept constant in the LES with and without radiation. Hence the mass fluxes differ, which is reflected in differences of $R e_{m}$. The fact that these differences are small (see table 1) already points towards a fairly weak effect of thermal radiation in the present computations. Please note that we use the standard definitions common in compressible turbulent flow, namely an overbar for a Reynolds average and a tilde for a mass-weighted average. Usually density/pressure/shear stress and velocity/ temperature/internal energy are decomposed as follows:

$$
\rho=\bar{\rho}+\rho^{\prime}, \quad u_{i}=\tilde{u_{i}}+u_{i}^{\prime \prime} .
$$

It may sometimes be necessary to use the Reynolds decomposition for the velocity, e.g. when it appears in a product with the pressure or the viscous stress. 


$\begin{array}{lccc}\text { Flow } & \begin{array}{c}\text { Domain size } \\ \text { in }(x, y, z) \text { direction }\end{array} & \begin{array}{c}\text { No. of points } \\ \text { in }(x, y, z) \text { direction }\end{array} & \begin{array}{c}\text { Non-dimensional grid spacing } \\ \text { in }(x, y, z) \text { direction }\end{array} \\ \text { Channel 1 } & 3.5 H \times 2 H \times 1.35 H & 96 \times 141 \times 64 & 37,2.1,21 \\ \text { Channel 2 } & 3.5 H \times 2 H \times 1.35 H & 96 \times 141 \times 64 & 50,3,29\end{array}$

TABLE 2. Details of computational domain and Cartesian grid in the $(x, y, z)$ direction.

Besides the friction Reynolds and Mach numbers, table 1 also contains the corresponding bulk Reynolds and Mach numbers for reference which are defined using the cross sectionally averaged (bulk) mean density and velocity, $\bar{\rho}_{m}$ and $\bar{u}_{m}$ :

$$
R e_{m}=\frac{\bar{\rho}_{m} \bar{u}_{m} H}{\mu_{w}}, \quad M_{m}=\frac{\bar{u}_{m}}{\sqrt{\gamma R T_{w}}}
$$

The last column contains values of the optical thickness computed via the statistically averaged Planck mean absorption coefficient (2.9):

$$
\tau_{H}=\int_{0}^{H} \bar{\kappa}_{P}(y) \mathrm{d} y .
$$

For very small values of $\tau_{H}$, a linear relation holds between the fraction of radiated energy that is absorbed and the optical thickness. Such a relation can be derived, starting from the definition of absorptivity $\alpha_{\eta}=1-\exp \left(-\int_{0}^{H} \kappa_{\eta} \mathrm{d} y\right)$ (Modest 2003). For a constant absorption coefficient the absorptivity depends on the thickness of the gas layer. Replacing $\kappa_{\eta}$ by $\bar{\kappa}_{P}$ and assuming $\bar{\kappa}_{P}$ and $H$ to be small, provides the desired linear relation. Hence $\tau_{H}=0.006$ indicates that about $0.6 \%$ of the energy emitted at the channel centre is absorbed by water vapour within the optical path travelled by photons from the channel centre to the wall.

Table 2 describes the size of the computational domain in streamwise $(x)$-, wallnormal $(y)$ - and spanwise $(z)$-direction, the number of gridpoints and the grid spacing in terms of semi-local wall units $\bar{\mu} /\left(\bar{\rho} \sqrt{\bar{\tau}_{w} / \bar{\rho}}\right)$. Values 2.1 and 3 of the dimensionless wall-normal grid spacing refer to the first near-wall gridpoint.

The mass, momentum and total energy (or pressure, temperature and velocity) in the minimal channel satisfy periodic boundary conditions in the streamwise and spanwise directions. In these planes, diffusely reflecting boundaries (with zero emissivity) are assumed in the radiative code, which ensure statistical homogeneity of the radiation fields in the homogeneous directions. At the solid walls all velocity components vanish, the temperature is kept at $1000 \mathrm{~K}$ in all computations and the emissivity is that of a black body, $\epsilon_{w}=1$.

\section{Mean flow variables and second-order turbulence statistics}

\subsection{Transport of momentum, energy and Reynolds stresses}

Reynolds or Favre averages are obtained by sampling the LES data in time and over the two homogeneous directions $(x$ and $z$ ). The simulations without radiation were performed over 180 non-dimensional times, $H / u_{\tau}$, on 96 processors of the SGI Altix 4700 high-performance computer of the Leibniz Supercomputing Centre of the Bavarian Academy of Sciences in Munich. One hundred and twenty non-dimensional times out of these were used to collect statistics at each point of the flow field. At the end of this time series, LES was fully coupled with the radiation solver to 
compute another 180 non-dimensional times of the flow field influenced by radiation. One thousand samples of this time series were used for time-averaging. A coupled simulation on 96 processors needed $276480 \mathrm{CPU}$ h when the SNB-cK spectral model was used. A simulation without radiation was faster by a factor of 15 .

The following mean flow equations look very similar to those derived by Huang, Coleman \& Bradshaw (1995), except for the appearance of the heat flux by radiation. Subgrid-scale terms do not appear in our equations, since we use an explicit filtering approach to account for the energy transfer at the cutoff wavenumber. The equations for mean streamwise and wall-normal mass flux in statistically steady and fully developed channel flow read, after integration in wall-normal direction and normalization by wall units, $u_{\tau}=\sqrt{\bar{\tau}_{w} / \bar{\rho}_{w}}, \mu_{w} /\left(\bar{\rho}_{w} u_{\tau}\right)$ :

$$
\begin{gathered}
\frac{\bar{\mu}}{\mu_{w}} \frac{\mathrm{d} \bar{u}^{+}}{\mathrm{d} y^{+}}-\frac{\overline{\rho u^{\prime \prime} v^{\prime \prime}}}{\bar{\tau}_{w}}=1-\frac{y^{+}}{H^{+}}, \\
\frac{\bar{p}}{\bar{p}_{w}}=1+\frac{\bar{\tau}_{y y}-\left.\bar{\tau}_{y y}\right|_{w}-\overline{\rho v^{\prime \prime} v^{\prime \prime}}}{\bar{p}_{w}} \simeq 1-\gamma M_{\tau}^{2} \frac{\overline{\rho v^{\prime \prime} v^{\prime \prime}}}{\bar{\tau}_{w}} .
\end{gathered}
$$

$y^{+}=\bar{\rho}_{w} y u_{\tau} / \mu_{w}$ is the so-called wall coordinate. Equation (4.1) expresses the fact that the sum of mean viscous stress and Reynolds stress varies linearly across the channel. Equation (4.2) contains a contribution from the mean wall-normal viscous stress which, however, turns out to be negligibly small. In both equations correlations involving viscosity fluctuations have been neglected. This is an acceptable assumption for supersonic flows (see Huang et al. 1995), but perhaps not for hypersonic flows. It will also be applied to conductive heat fluxes below. The second term on the right-hand side of (4.1) results from the driving pressure gradient and contains the friction Reynolds number in the denominator, $H^{+}=R e_{\tau}$. While the mean pressure has a streamwise gradient which is homogeneous everywhere in the channel, its wallnormal variation follows approximately the variation of the wall-normal Reynolds stress (4.2) and is much smaller than the variation of mean density and temperature. Hence mean density and temperature vary inversely with each other.

For comparison of the streamwise velocity with its incompressible counterpart in the $\log$ region, it is usual to present it in its Van Driest transformed version:

$$
\bar{u}_{V D}^{+}=\int_{0}^{\bar{u}^{+}} \sqrt{\frac{\bar{\rho}}{\bar{\rho}_{w}}} \mathrm{~d} \bar{u}^{+} .
$$

To discuss the importance of the radiative heat flux, $\bar{q}_{y, R}$, compared to the other heat fluxes, we start from the mean total energy balance and integrate it from the wall to a point $y$ below the centreline. This leads to

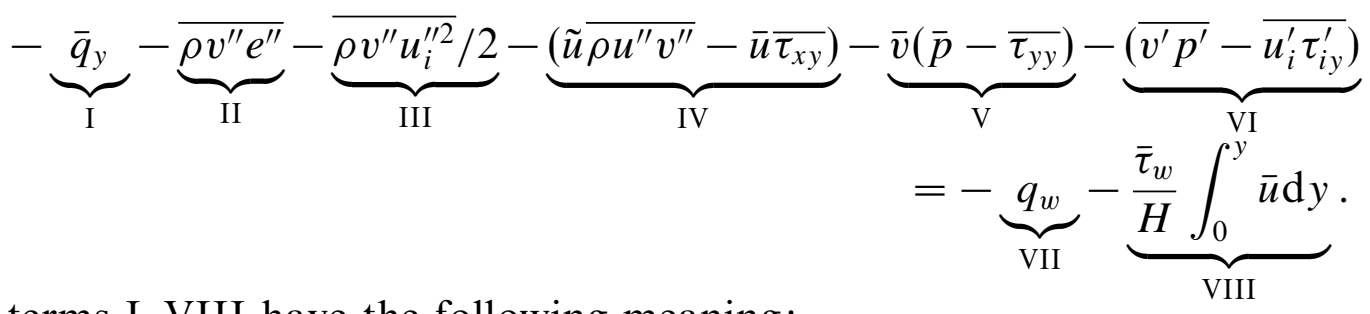

The terms I-VIII have the following meaning:

I: heat flux by conduction and radiation;

II: turbulent heat flux;

III: turbulent transport of kinetic energy;

IV: work done by viscous and Reynolds stresses; 
$\mathrm{V}$ : work done by mean flow in wall-normal direction;

VI: work done by turbulent fluctuations;

VII: wall heat flux by conduction and radiation;

VIII: pressure work done between the wall and position $y$.

Concerning term $\mathrm{V}$ one must keep in mind that it is very small, because $\bar{v}$ is very small. While the mass-averaged normal velocity, $\tilde{v}$, is zero in fully developed channel flow, the Reynolds averaged velocity is proportional to the weak wall-normal turbulent mass-flux, $\bar{v}=-\overline{v^{\prime \prime}}=-\overline{\rho^{\prime} v^{\prime}} / \bar{\rho}$.

On the centreline all terms on the left-hand side of (4.4) vanish due to symmetry and we obtain

$$
q_{w}=-\left[\bar{\lambda} \frac{\mathrm{d} \bar{T}}{\mathrm{~d} y}\right]_{w}+q_{w, R}=-\frac{\bar{\tau}_{w}}{H} \int_{0}^{H} \bar{u} \mathrm{~d} y=-\bar{\tau}_{w} \bar{u}_{m}=H \frac{\partial \bar{p}}{\partial x} \bar{u}_{m} .
$$

This relation expresses the fact that the sum of conductive and radiative heat fluxes into the wall equals the total mechanical work done in the channel by the mean pressure. Hence, the right-hand side of (4.4) can also be cast in the following form:

$$
-q_{w}-\frac{\bar{\tau}_{w}}{H} \int_{0}^{y} \bar{u} \mathrm{~d} y=\frac{\bar{\tau}_{w}}{H} \int_{y}^{H} \bar{u} \mathrm{~d} y
$$

Taking into account that the heat flux at a distance $y$ from the wall is the sum of conductive and radiative fluxes, $\bar{q}_{y}=-\bar{\lambda}(\mathrm{d} \bar{T} / \mathrm{d} y)+\bar{q}_{y, R}$, we obtain the following balance equation:

$$
\begin{gathered}
\underbrace{\bar{\lambda} \frac{\mathrm{d} \bar{T}}{\mathrm{~d} y}-\bar{q}_{y, R}}_{\mathrm{I}}-\underbrace{\overline{\rho v^{\prime \prime} e^{\prime \prime}}}_{\mathrm{II}}-\underbrace{\overline{\rho v^{\prime \prime} u_{i}^{\prime \prime 2}} / 2}_{\mathrm{III}}-\underbrace{\left(\tilde{u} \overline{\rho u^{\prime \prime} v^{\prime \prime}}-\bar{u} \overline{\tau_{x y}}\right.}_{\mathrm{IV}})-\underbrace{\bar{v}\left(\bar{p}-\overline{\tau_{y y}}\right)}_{\mathrm{V}}-\underbrace{\left(\overline{v^{\prime} p^{\prime}}-\overline{u_{i}^{\prime} \tau_{i y}^{\prime}}\right)}_{\mathrm{VI}} \\
=\underbrace{\frac{\bar{\tau}_{w}}{H} \int_{y}^{H} \bar{u} \mathrm{~d} y}_{\mathrm{VII}+\mathrm{VIII}} .
\end{gathered}
$$

This equation must, in principle, be integrated in $y$-direction, in order to allow for conclusions concerning the effect of radiation on the distribution of the mean temperature. In its present form, however, (4.7) already presents valuable insight, which will be discussed in $\S \S 4.2$ and 4.3 .

The transport of Reynolds stresses, $\overline{\rho u_{i}^{\prime \prime} u_{j}^{\prime \prime}}$, is controlled in fully developed channel flow by the following balance equation:

$$
\begin{aligned}
0= & \underbrace{-\overline{\rho u_{j}^{\prime \prime} v^{\prime \prime}} \frac{\mathrm{d} \tilde{u}}{\mathrm{~d} y} \delta_{i x}-\overline{\rho u_{i}^{\prime \prime} v^{\prime \prime}} \frac{\mathrm{d} \tilde{u}}{\mathrm{~d} y} \delta_{j x}}_{P_{i j}} \underbrace{-\frac{\mathrm{d}}{\rho d y} \overline{\rho u_{i}^{\prime \prime} u_{j}^{\prime \prime} v^{\prime \prime}}}_{P T_{i j}} \underbrace{-\frac{\mathrm{d}}{\mathrm{d} y}\left(\overline{u_{i}^{\prime} p^{\prime}} \delta_{j y}-\overline{u_{j}^{\prime} p^{\prime}} \delta_{i y}\right)}_{P D_{i j}} \\
& \underbrace{+\frac{\mathrm{d}}{\mathrm{d} y}\left(\overline{u_{i}^{\prime} \tau_{j k}^{\prime}}+\overline{u_{j}^{\prime} \tau_{i k}^{\prime}}\right) \delta_{k y}}_{V D_{i j}} \underbrace{-\overline{u_{i}^{\prime \prime}}\left(\frac{\partial \bar{p}}{\partial x_{j}}-\frac{\mathrm{d} \overline{\tau_{j y}}}{\mathrm{~d} y}\right)-\overline{u_{j}^{\prime \prime}}\left(\frac{\partial \bar{p}}{\partial x_{i}}-\frac{\mathrm{d} \overline{\tau_{i y}}}{\mathrm{~d} y}\right)}_{M_{i j}} \\
& \underbrace{\overline{p^{\prime}\left(\frac{\partial u_{i}^{\prime}}{\partial x_{j}}+\frac{\partial u_{j}^{\prime}}{\partial x_{i}}\right)} \underbrace{\overline{\frac{\partial u_{i}^{\prime}}{\partial x_{k}}}-\overline{\tau_{i k}^{\prime} \frac{\partial u_{j}^{\prime}}{\partial x_{k}}}}_{-\overline{\tau_{j k}^{\prime}}} .}_{D S_{i j}}
\end{aligned}
$$

The labels of the various tensors in this equation have the following meaning: $P_{i j}$ : production, $T T_{i j}$ : turbulent transport, $P D_{i j} / V D_{i j}$ : pressure and viscous diffusion, 

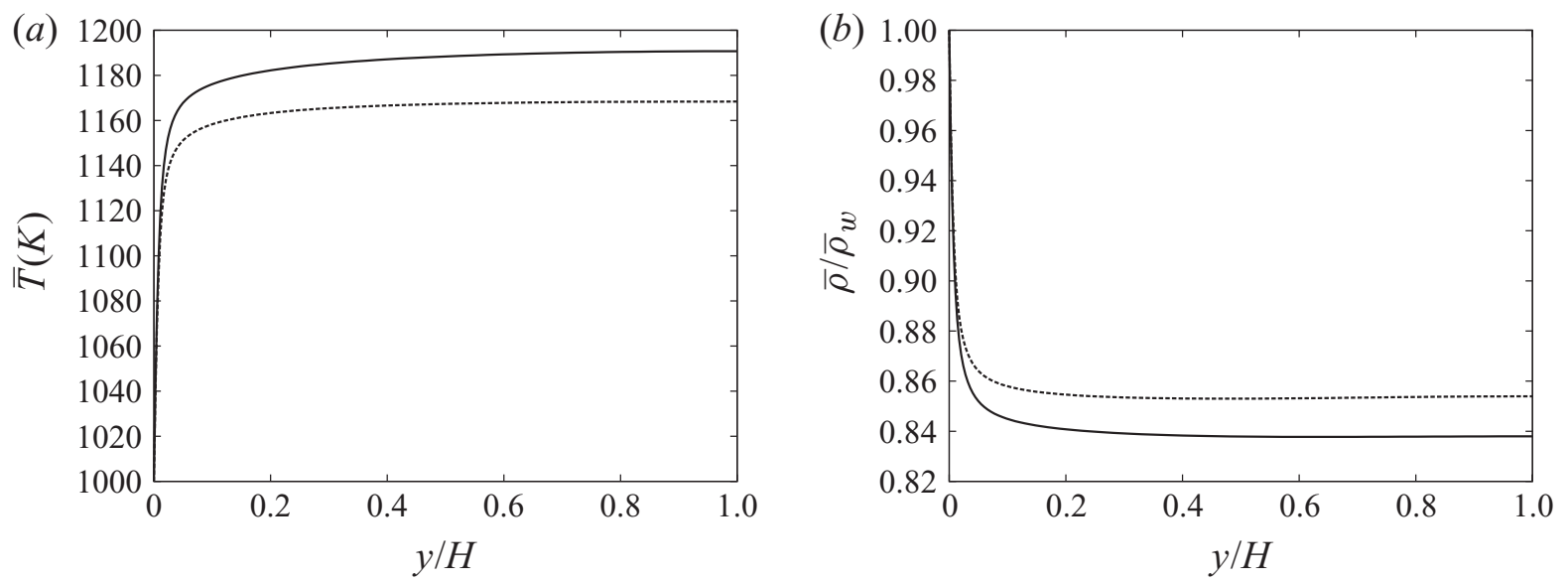

FIgURE 2. Mean temperature $(a)$ and mean density distribution $(b)$. Dashed line: with radiation, solid line: without radiation.

$M_{i j}$ : mass-flux contribution, $P S_{i j}$ : pressure-strain correlation and $D S_{i j}$ : viscous or turbulent dissipation. Thermal radiation can, of course, affect the Reynolds stress transport only implicitly, and it will be interesting to learn through which terms this will happen. From previous comparisons of DNS and LES of the Reynolds stress transport in pipes Ghosh et al. (2006), we conclude that the production and the pressure-strain correlation terms are predicted in our LES with sufficient accuracy, whereas the dissipation term lacks the contribution of the unresolved small scales. This, however, is not a critical issue, since our aim is primarily a comparison of flow with and without radiation effects at very similar Reynolds and Mach numbers, in order to detect trends of the effect of radiation. Moreover, the smallest (unresolved) turbulent scales will be optically transparent to photons in the flow cases investigated and hence may not contribute to TRI.

\subsection{Case of low supersonic Mach number}

Before we contrast the two flow cases, we first analyse the influence of radiation on turbulent channel flow at a low supersonic Mach number, where the temperature increase in the channel core is roughly $20 \%$ of the wall temperature (case: Channel 1 ). We assume the fluid, pure water vapour, to be an emitting/absorbing but nonscattering medium and recall the characteristics of the flow: a bulk Mach number of 1.26, a friction Reynolds number of 1026 and a mean optical thickness of 0.006 . The wall temperature is kept at $1000 \mathrm{~K}$ in the cases with and without radiation. The medium appears optically 'thicker' than that of Channel 2 because, in order to achieve comparable Reynolds numbers, the lower mass flux of Channel 1 had to be multiplied by a value of $H$ which is higher than that of Channel 2. Moreover, the lower temperature level at Mach 1.26 leads to higher mean absorption coefficients than those at higher temperature (Gupta et al. 2009).

As expected, the effect of radiation is to decrease the mean temperature in the channel core compared to its value when radiation is turned off (figure $2 a$, solid line). The near-wall rise in mean temperature from $1000 \mathrm{~K}$ at the wall to $1190 \mathrm{~K}$ in the core region is due to kinetic energy dissipation. As a result of emission and absorption of radiated heat between the channel centreline and the wall, the temperature in the core is reduced by about $20 \mathrm{~K}$ (figure $2 a$, dashed line). Consequently, the mean density (figure $2 b$ ) falls off more gently from its wall value, is higher in the core region and, as discussed above, varies inversely with the mean temperature. 

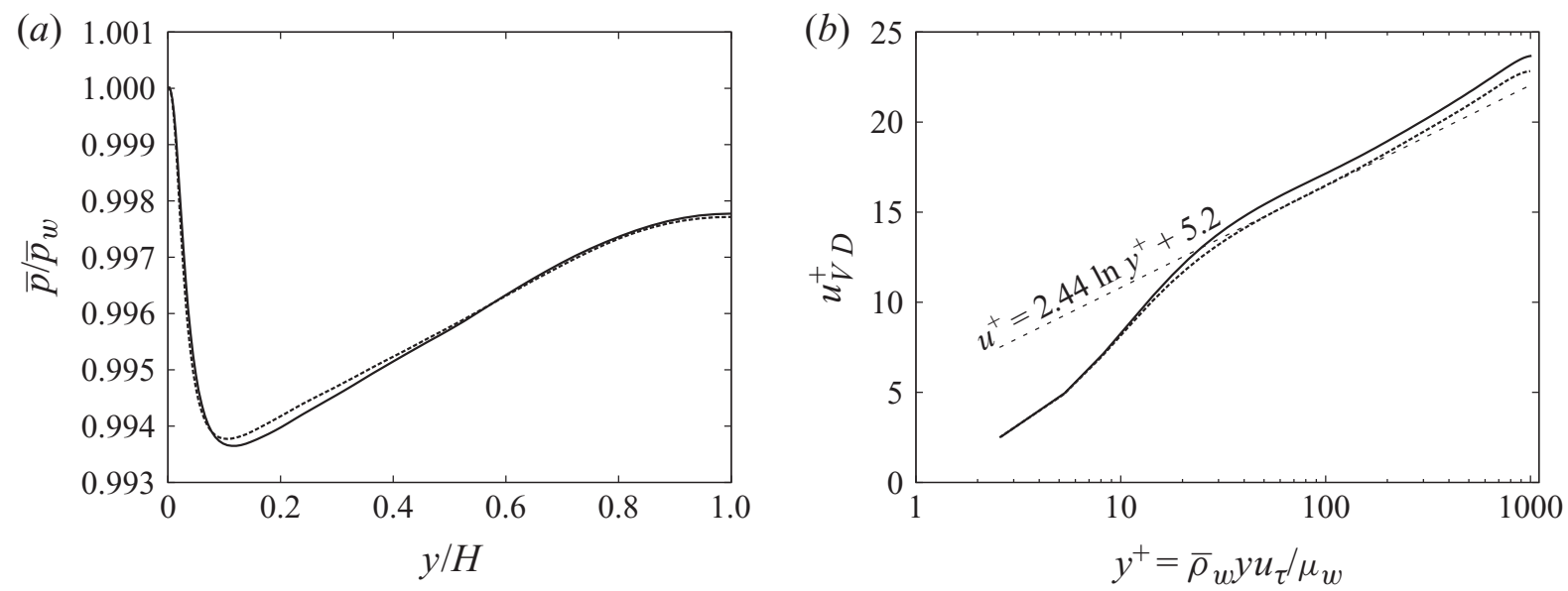

FIGURE 3. Mean pressure distribution ( $a$ ), mean streamwise, Van Driest transformed velocity $(b)$. Lines as in figure 2.

Using the averaged thermal equation of state and its form in the immediate vicinity of the wall,

$$
\bar{p}=\bar{\rho} R \bar{T}+R \overline{\rho^{\prime} T^{\prime}}, \quad \bar{p}_{w}=\bar{\rho}_{w} R T_{w},
$$

and considering that the wall-normal variations of the density-temperature correlation and of the mean pressure are small compared to corresponding variations of $\bar{\rho}, \bar{T}$, we obtain

$$
\frac{1}{\bar{\rho} / \bar{\rho}_{w}} \frac{\mathrm{d} \bar{\rho} / \bar{\rho}_{w}}{\mathrm{~d} y} \simeq-\frac{1}{\bar{T} / T_{w}} \frac{\mathrm{d} \bar{T} / T_{w}}{\mathrm{~d} y}
$$

Hence, the normalized mean density and temperature gradients are practically equal in magnitude, but opposite in sign. Figure 3 presents the effect of radiation on the mean pressure, $\bar{p}$, and the mean streamwise velocity, $\bar{u}$, which we conveniently plot in its Van Driest transformed version, (4.3), against the wall coordinate. The log-law, $u^{+}=2.44 \ln y^{+}+5.2$, is also plotted for reference. Let us now look at the effect of thermal radiation on second-order moments and start with the variances of density and temperature fluctuations or their r.m.s. values, which we plot against the semi-local coordinate $y^{*}=\bar{\rho} y \sqrt{\bar{\tau}_{w} / \bar{\rho}} / \bar{\mu}$. While the normalized mean density increase in the channel core, compared to the flow without radiation, is less than $2 \%$ of the local value (figure 2), the decrease in the r.m.s. density fluctuations, normalized by the local mean density, reaches $18 \%$ and more in the log layer (figure $4 a$ ). It was shown by Lechner, Sesterhenn \& Friedrich (2001) that the production of density variances in fully developed channel flow is only by mean density gradients and not by mean dilatation, since the flow is neither accelerated nor retarded in the streamwise direction. Hence, the reduction in $\rho_{r m s} / \bar{\rho}$ is consistent with the increase of $\bar{\rho} / \bar{\rho}_{w}$. Similar arguments hold for the r.m.s. temperature fluctuations in figure $4(b)$, which are reduced by about $25 \%$ in the log layer, due to thermal radiation, since the mean temperature gradients are smaller across the channel. For completeness we also present the r.m.s. pressure fluctuations, normalized with the wall-shear stress, in figure 5. Here, a similar observation is made, namely a reduction of the order of $10 \%$ due to radiation.

Plots of each of the four Reynolds stresses in figures 6 and 7 show that radiation counteracts effects of compressibility. In the following we will explain what we mean by this. It was first discussed by Coleman, Kim \& Moser (1995) and Huang et al. (1995) that compressibility manifests itself in terms of mean property variations, i.e. in 

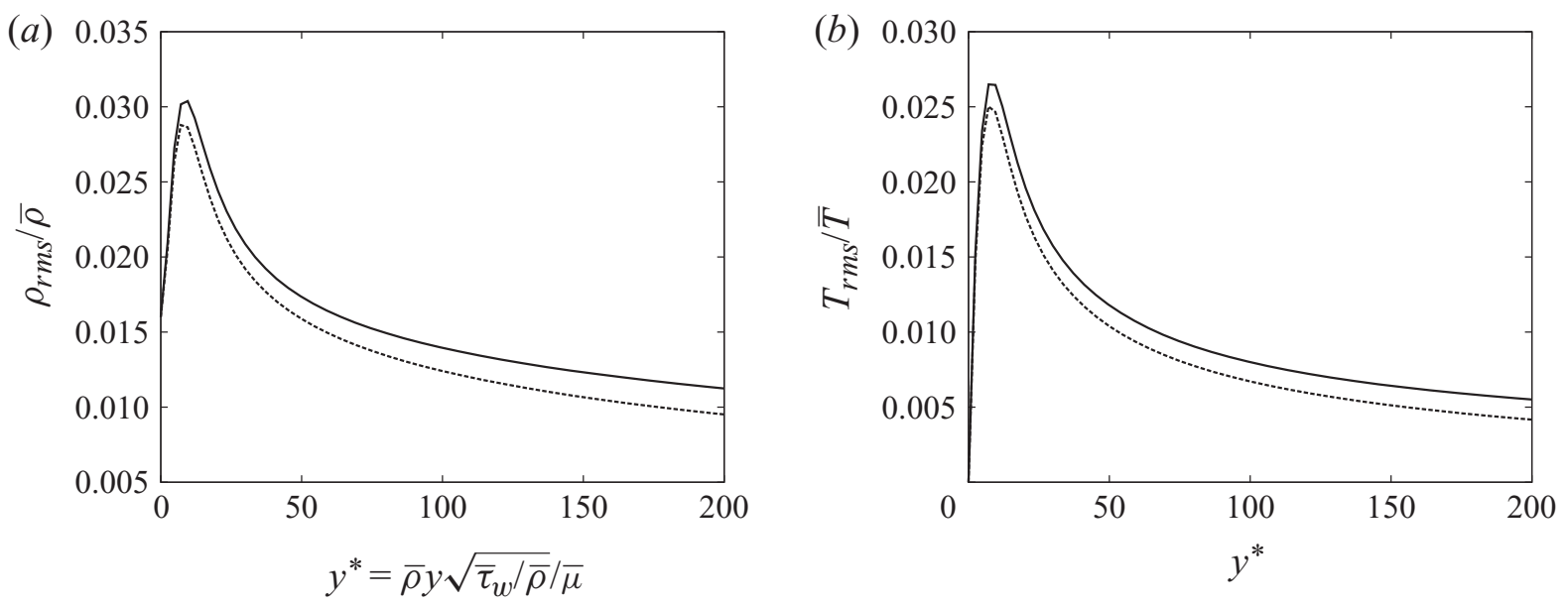

FIGURE 4. RMS density (a) and temperature (b) fluctuations. Lines as in figure 2.

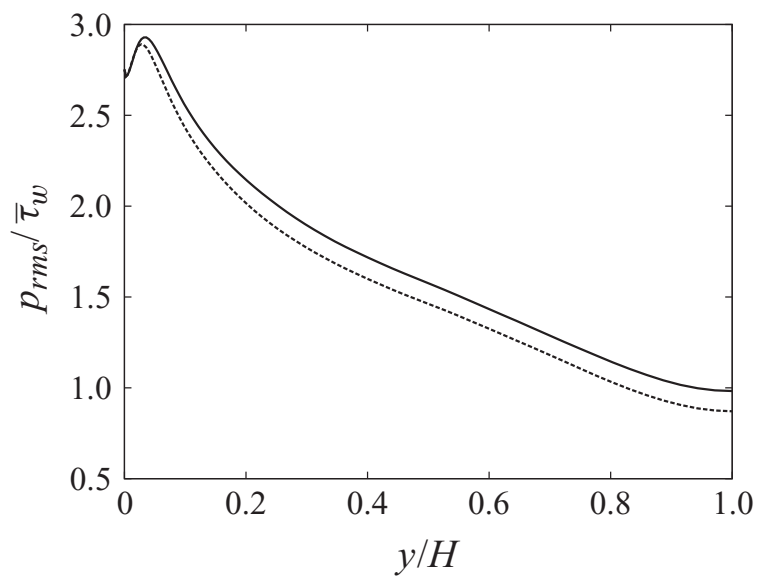

FIGURE 5. RMS pressure fluctuations. Lines as in figure 2.
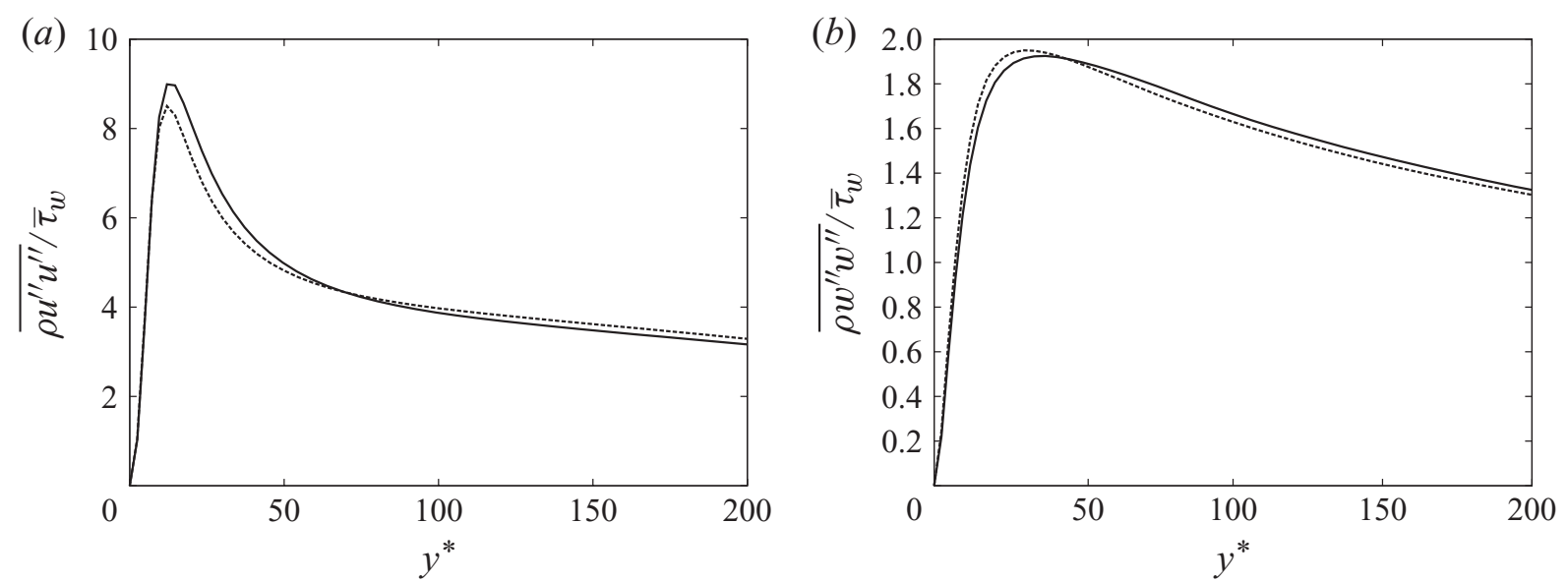

Figure 6. Streamwise $(a)$ and spanwise $(b)$ Reynolds stresses. Lines as in figure 2.

the decrease of mean density and increase of mean viscosity from wall values. Later Foysi, Sarkar \& Friedrich (2004) provided an explanation for the decrease in the pressure-strain correlations and increase in the Reynolds stress anisotropy, compared to incompressible flow. They found, using a Green's function analysis of the pressure fluctuations and computing the pressure-strain correlation tensor as a convolution integral, that the fall-off of mean density from its wall value causes a decrease of 

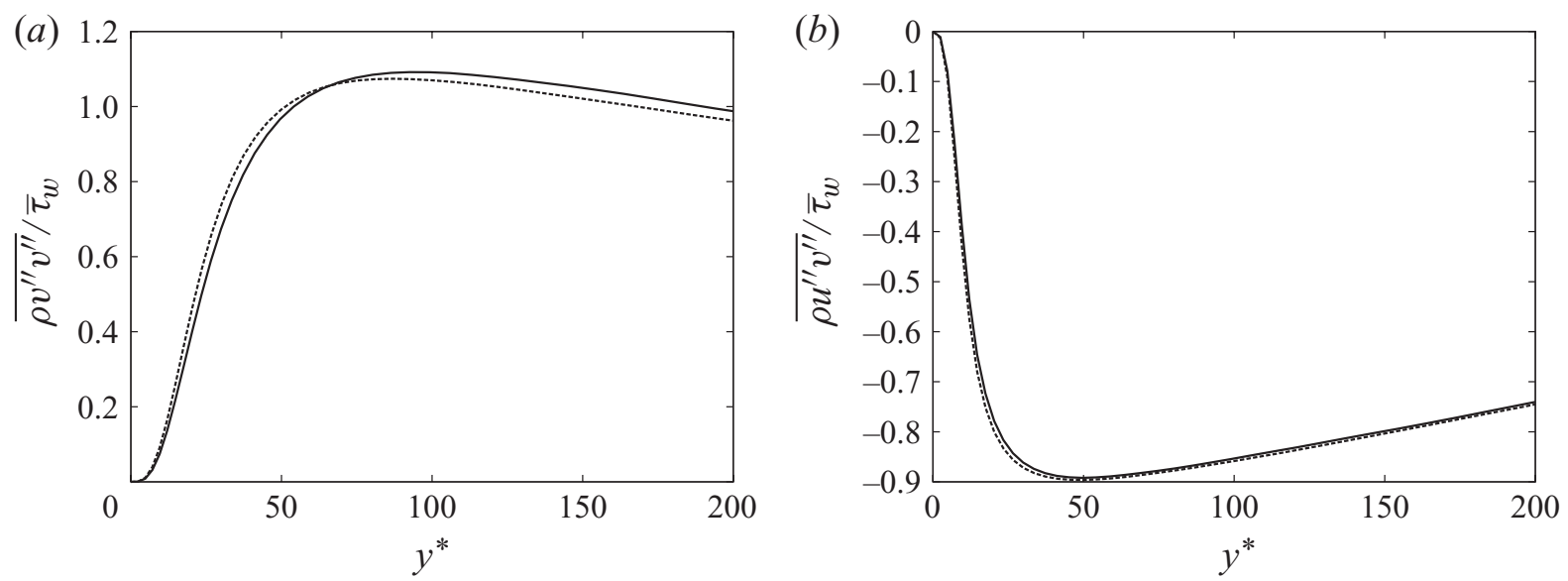

FigURE 7. Wall-normal Reynolds stress $(a)$ and Reynolds shear stress $(b)$. Lines as in figure 2.

the pressure-strain correlation tensor compared to its incompressible counterpart. A reduced pressure-strain correlation in the streamwise Reynolds stress balance (while production does not change noticeably) means that less kinetic energy is transferred to the other normal Reynolds stresses via redistribution, so that the streamwise stress increases in the wall layer compared to the incompressible case, while all other stresses obtain less energy and hence show reduced amplitudes there. Following the same line of argument, we conclude that an increase in mean density compared to the case without thermal radiation (figure $2 b$ ) will reduce the streamwise Reynolds stress and increase the other three stress components in the wall layer, where the production of the turbulence kinetic energy and the pressure-strain correlations for the streamwise and spanwise Reynolds stress balances peak $\left(0 \leqslant y^{*} \leqslant 50\right)$. This behaviour is indeed observed, although changes in amplitudes are not very pronounced (see figures 6 and 7). The Reynolds shear stress in figure $7(b)$ is even slightly increased everywhere, which is consistent with the decrease in the mean velocity.

It is worth noting that the behaviour of the mean pressure, as predicted by (4.2), is also consistent with that of the wall-normal Reynolds stress, since the turbulent Mach number shows a negligible variation due to radiation. Hence, a local decrease in the mean pressure is the consequence of a local increase in the wall-normal Reynolds stress and vice versa.

We now present the effect of radiation on the behaviour of two terms of the pressure-strain correlation tensor $\left(P S_{i j}\right)$, normalized with the wall-shear stress squared divided by the local mean viscosity, in order to provide physical support for the implicit effect of radiation on the corresponding Reynolds stresses. Figure 8 contains profiles of the normalized terms $P S_{x x}$ and $P S_{z z}$, for the cases with and without radiation. All dashed curves show larger amplitudes in the wall layer as a result of a mean density increase by radiation. This translates into an enhanced redistribution mechanism, meaning that the streamwise Reynolds stress is reduced in the wall layer, because $P S_{x x}$ (being negative) takes more energy out of this component and transfers it to the other two components. $P S_{z z}$ has more energy in the case with radiation than without radiation and hence causes the spanwise stress to increase. This reasoning comprises the assumption that the production term, appearing in the balance equation (4.8) for the streamwise Reynolds stress, normalized in the same way as $P S_{x x}$, is hardly affected by radiation. Figure $9(a)$ indeed confirms this assumption.

Finally, we display the important terms of the heat flux balance in figure $9(b)$, normalized by the total heat flux at the wall (4.7). These terms are, using the labels 

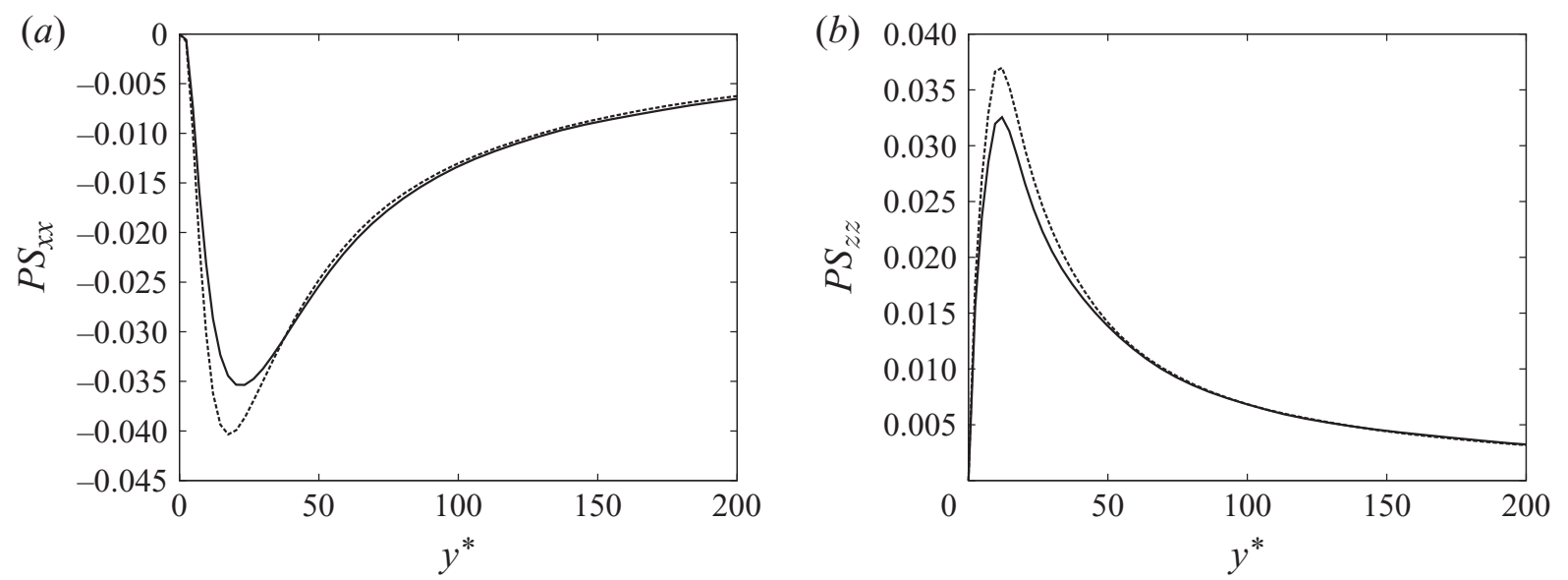

FiguRE 8. Pressure-strain correlations $P S_{x x}(a)$ and $P S_{z z}(b)$ normalized with $\bar{\tau}_{w}^{2} / \bar{\mu}$. Lines as in figure 2.
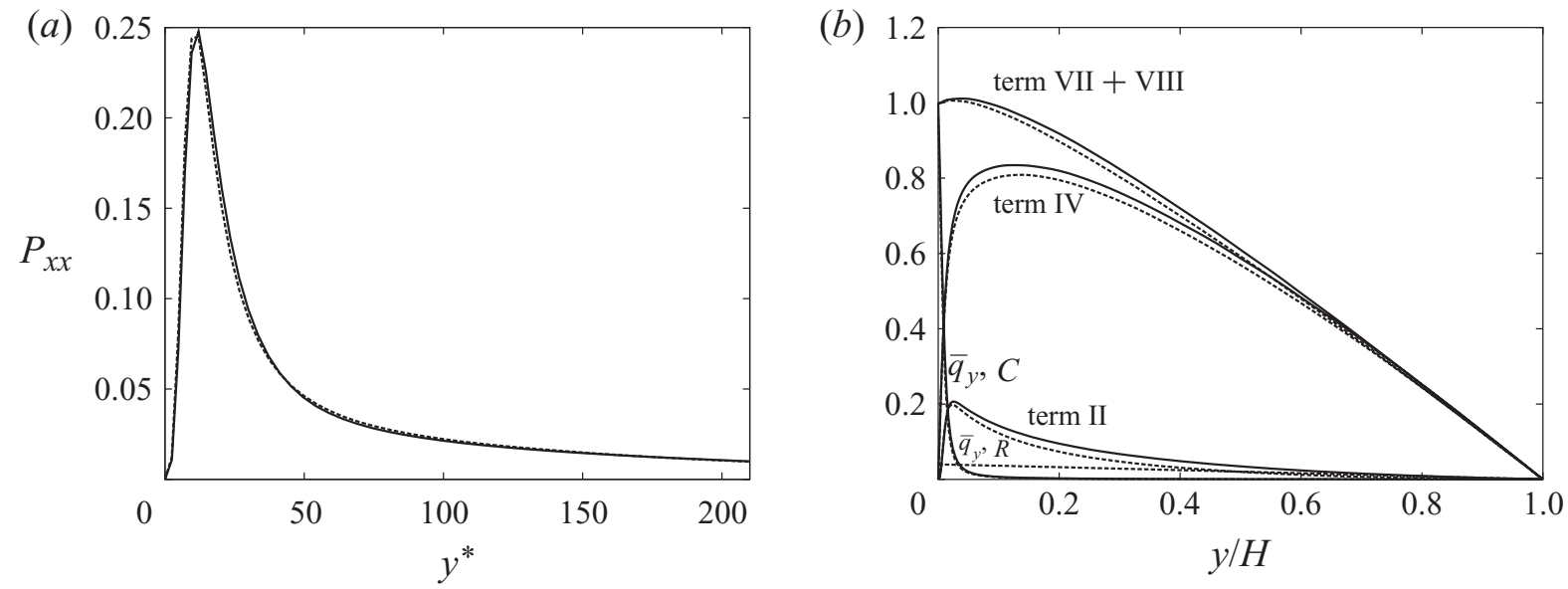

FiguRE 9. Production term $P_{x x}(a)$ normalized with $\bar{\tau}_{w}^{2} / \bar{\mu}$. Heat flux balance (4.7) in Channel 1 scaled by the total heat flux at the wall $(b)$. Lines as in figure 2.

of (4.7), the heat fluxes by conduction and radiation (term I), $\bar{q}_{y, C}, \bar{q}_{y, R}$, the turbulent heat flux (term II), the work done by the sum of viscous and Reynolds shear stresses (term IV) and the pressure work done between position $y$ and the centreline (terms VII + VIII). Starting with the pressure work (right-hand side of (4.7)), we note that the term corresponds to the total wall heat flux at $y=0$ and is zero at the centreline. It varies nonlinearly in between. The effect of radiation (dashed curve) is fairly weak, but still clearly visible. This is also true for the heat flux by conduction, which amounts to $96 \%$ of the total heat flux at the wall. To show this more clearly, it is convenient to display the terms only in the wall layer versus the semi-local coordinate (see figure 10). The heat flux by radiation is zero at the centreline for symmetry reasons (figure $9 b$ ) and increases roughly linearly towards the wall, since the optical thickness is quite low (Amaya et al. 2010). A large contribution to the heat flux in the wall layer and in parts of the channel core comes from the work done by viscous and Reynolds stresses (term IV). The effect of radiation is to decrease this flux slightly. The turbulent heat flux which peaks, where the turbulence production peaks, shows a decrease due to thermal radiation as well. The next section provides evidence that smaller optical thickness and a higher temperature level in the channel core decrease this coupling even more and underline that there is no need for fully coupled simulations. 


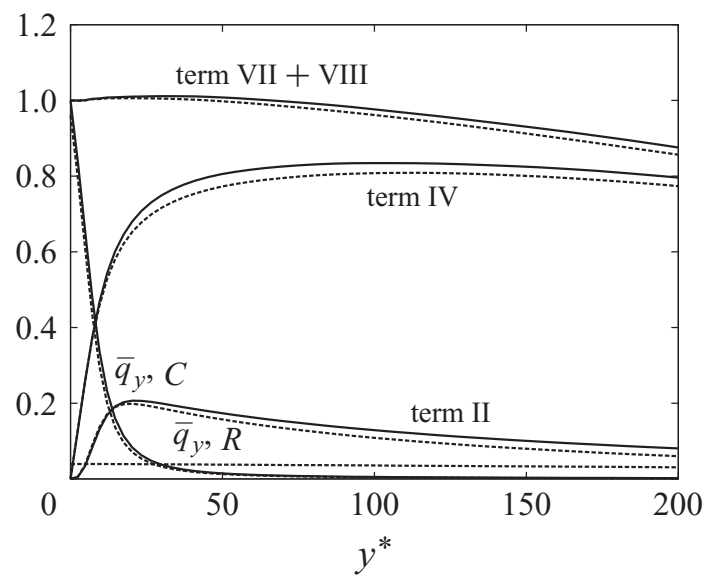

Figure 10. Heat flux balance (4.7) in Channel 1 scaled by the total heat flux at the wall. Lines as in figure 2.
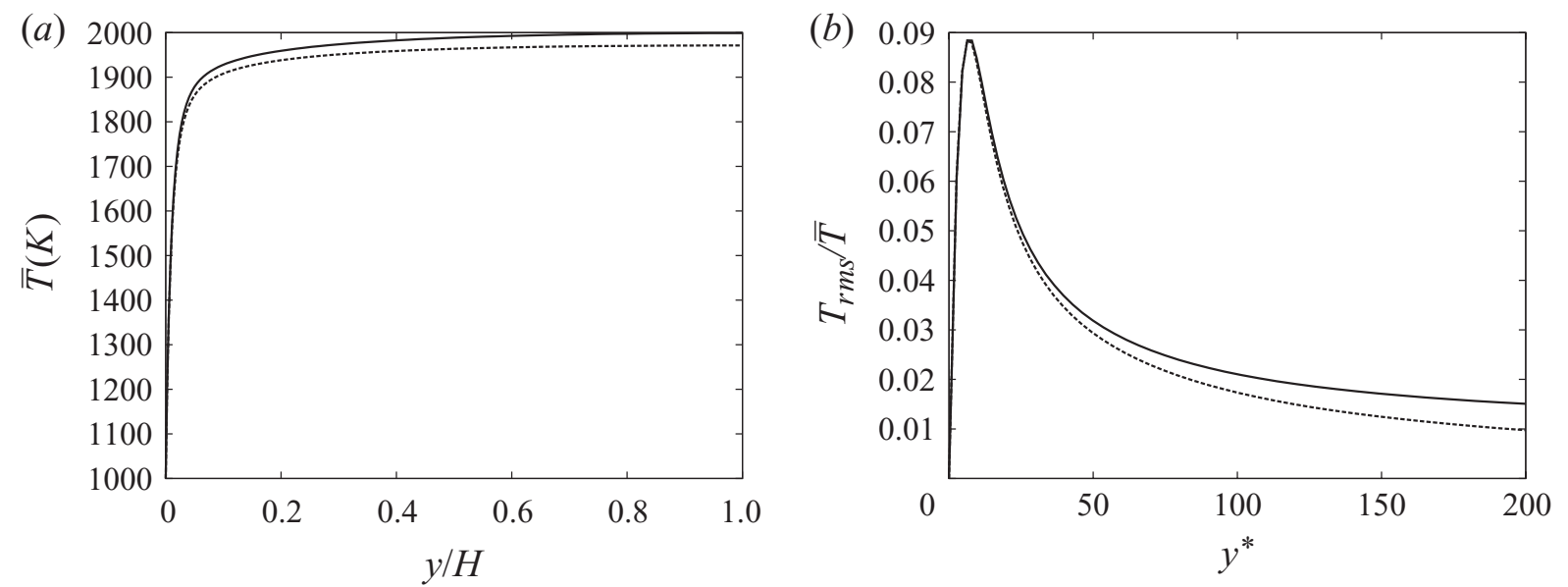

FIGURE 11. Mean temperature $(a)$ and r.m.s. temperature fluctuations $(b)$. Dashed line: with radiation, solid line: without radiation.

\subsection{Case of high supersonic Mach number}

The flow parameters for Channel 2, listed in table 1, reveal that an increase in friction Mach number or bulk Mach number entails an increase in friction Reynolds number, which, in principle, is not desired, if the aim is a discussion of effects of compressibility at equal Reynolds number. However, to keep the friction Reynolds number equal to that of Channel 1 while increasing the friction Mach number would involve an iterative process of successive LES with step-by-step changing parameters, which was not undertaken. In the present case, the optical thickness of the gas layer averaged over the channel half-width drops by a factor of 2 compared to that of Channel 1 . This is so, because the higher mass flux in Channel 2 implies a lower thickness of the gas layer (channel half-width) to keep the Reynolds number close to that of Channel 1. At the same time the absorption coefficient has lower values at higher temperatures. As figure 11(a) shows, kinetic energy dissipation raises the mean temperature steeply from $1000 \mathrm{~K}$ at the wall to $1998 \mathrm{~K}$ at the channel centreline, when radiation is turned off. Now, radiative transfer of energy brings it down to $1971 \mathrm{~K}$, which is a maximum difference of $27 \mathrm{~K}$. As a result of the strong temperature gradients the generation of temperature fluctuations is higher than in Channel 1, reaching a maximum value of $140 \mathrm{~K}$, and when normalized with the local mean temperature, reaching a peak value of 0.09 (figure 11b). Mean velocity and Reynolds stresses show a behaviour 

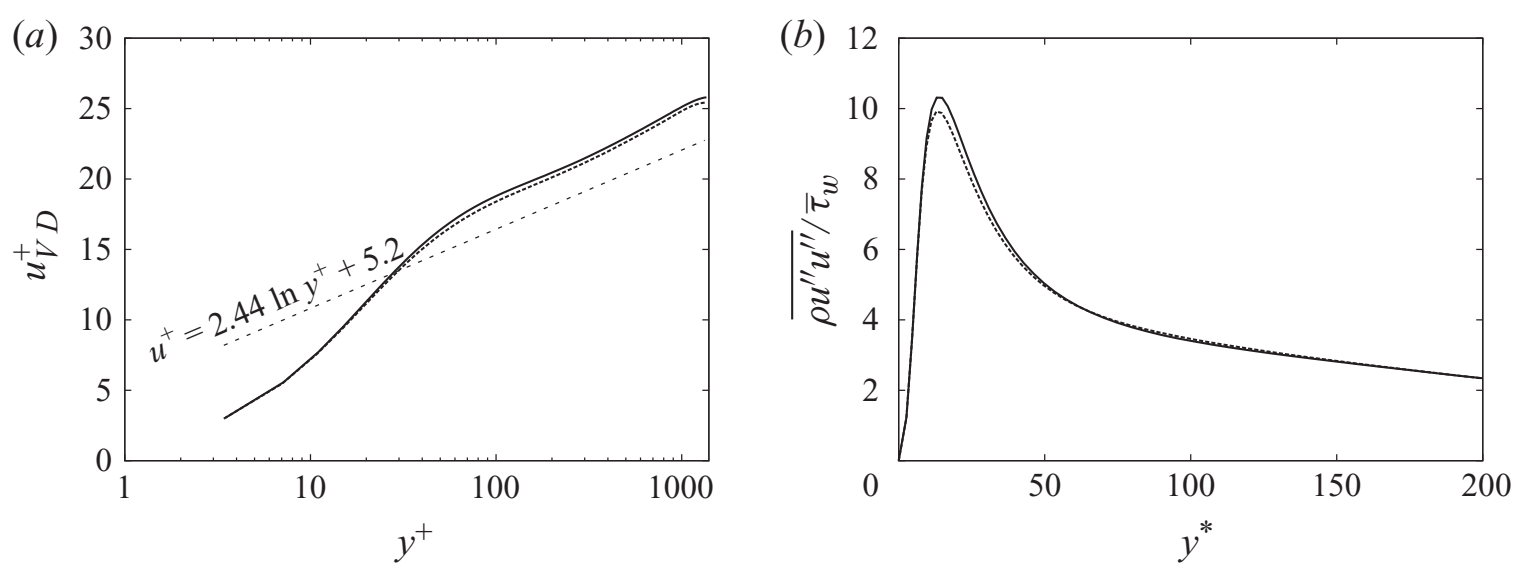

FIgURE 12. Van Driest transformed velocity $(a)$ and streamwise Reynolds stress $(b)$. Line types as in figure 11 .
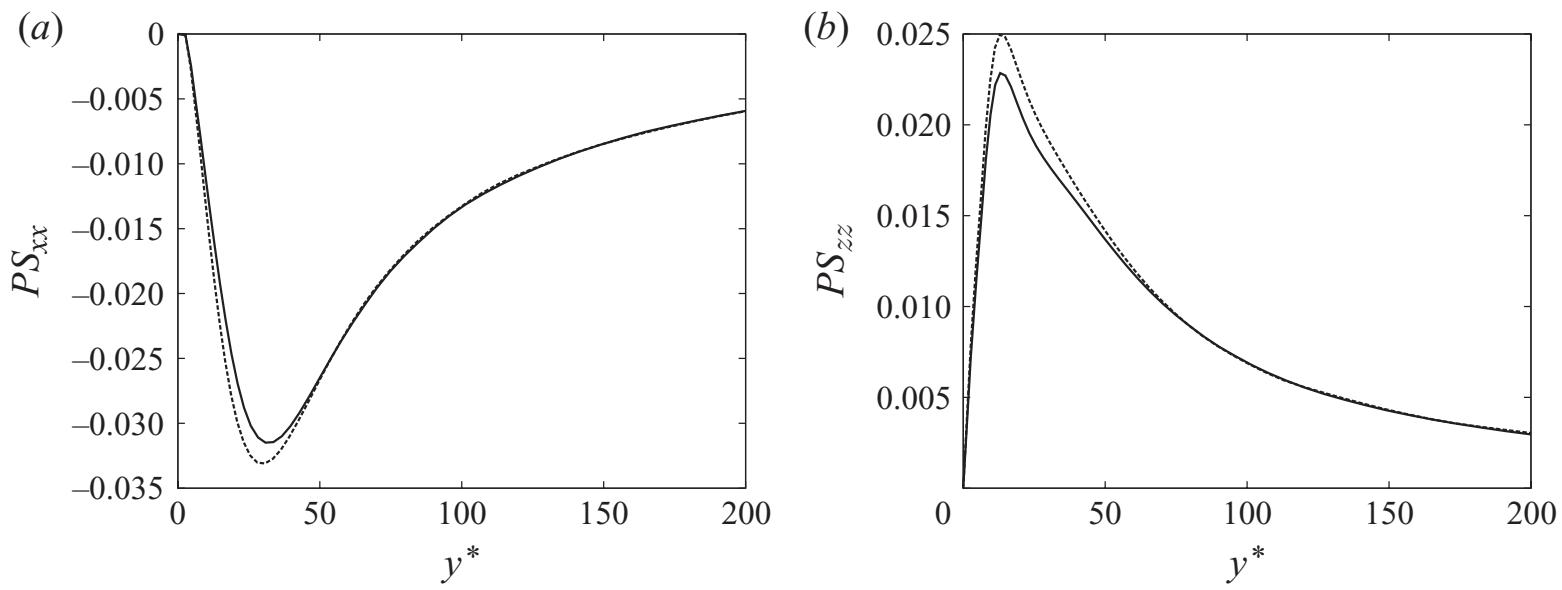

FIGURE 13. Normalized pressure-strain correlations, $P S_{x x}(a)$ and $P S_{z z}(b)$. Lines as in figure 11.

under the influence of radiation which is very similar to that in the lower Machnumber case, with the only difference that the effect of radiation is now considerably weaker than before (see figure 12). A well-known compressibility effect is that the Van Driest transformed velocity moves further away from the $\log \operatorname{law}, u^{+}=2.44 \ln y^{+}+5.2$, as the Mach number increases. This is quite obvious when figures $3(b)$ and $12(a)$ are compared. When we contrast the streamwise Reynolds stress in the present case (figure 12b) with that for Channel 1 (figure $6 a$ ), we observe an effect already discussed by Foysi et al. (2004), namely the increase in the peak value when the Mach number increases. Here, the higher Reynolds number (compared to Channel 1) adds to this effect. Radiation counteracts the effect of compressibility, in agreement with the lower Mach-number case. The other Reynolds stresses (not depicted here) show exactly the same behaviour as presented in figures 6 and 7, however with even smaller effects of radiation in the wall layer $\left(0 \leqslant y^{*} \leqslant 50\right)$. The extreme values of the pressure-strain correlations in figure 13 are smaller than those in Channel 1 due to the higher Mach numbers, although the Reynolds numbers are increased. This is a clear effect of compressibility, while the enhancement of these correlations in the wall layer (dashed curves) is a radiation effect.

We complete the discussion of Mach-number effects here by presenting important heat flux terms in Channel 2 (see figure 14). When comparing these terms with those for the lower Mach-number case (figure $9 b$ ), one obvious and noteworthy difference is 


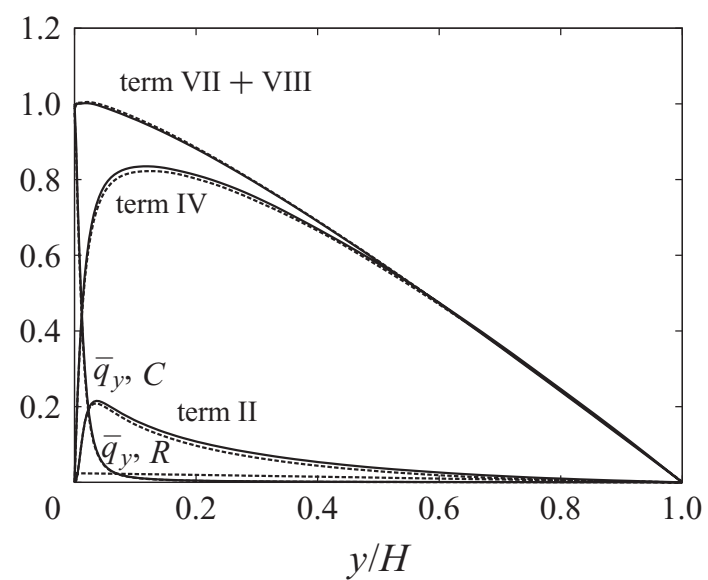

Figure 14. Heat flux balance (4.7) in Channel 2 scaled by the total heat flux at the wall. Lines as in figure 11.

that the heat flux by radiation is smaller, relative to the total heat flux at the wall and that, as a consequence, the remaining curves representing the flow with and without radiation are closer to each other than that for Channel 1 . Hence, in this specific case, there is no need for fully coupled simulations between compressible turbulent flow and thermal radiation, so that the radiative energy flux at the wall can be computed from the flow data using the radiation solver.

\subsection{Radiative energy emitted and absorbed across the channel}

We have seen how thermal radiation affects the structure of supersonic turbulent flow in a channel and how the interaction between turbulence and radiation decreases as the Mach number increases, partly because the absorption coefficient decreases at higher temperature levels and partly because the width of Channel 2 is smaller than that of Channel 1, in order to keep the Reynolds numbers roughly fixed.

Now we ask the question, how the energies which are emitted and absorbed by water vapour change between the channel walls when we contrast the two cases of table 1. Before answering this question we present a snapshot of the integrals on the right-hand side of (2.8) for Channel 1. The first of these integrals defines the energy emitted by the gas at a point $(x, y, z)$, whereas the second integral describes the absorbed energy at the same point. The difference between these two integrals corresponds to the instantaneous source term in the energy equation, which is the divergence of the radiative heat flux. This source term is not zero close to the wall, but it is quite small, corresponding to the weak gradient of the radiative heat flux (see figure 10). Figure 15( $a$ ) shows the instantaneous emission and absorption integrals in $1 / 8$ of the vertical plane at $z / H=0.675$.

In figure $15(b)$ we have plotted the instantaneous temperature field which is the reason for the emission and absorption integrals in figure 15(a). Although these integrals and the radiative properties of water vapour are nonlinear functions of temperature, we are able to make out certain characteristic flow structures in the emission and temperature fields. We also note that the amount of absorption in the channel is, on average, not more than $8 \%$ of emission at wall temperature. Figure 16 shows snapshots of emission/absorption and temperature in Channel 2 at $M_{m}=2.88$, again in $1 / 8$ of the vertical plane at $z / H=0.675$. The emission integral reaches values which are by a factor of 4 to 5 larger than at the wall, while the magnitude of the absorption integral is of the order of $10 \%$ of the emission integral at the wall. Again characteristic flow structures can be made out in the emission and temperature fields. 

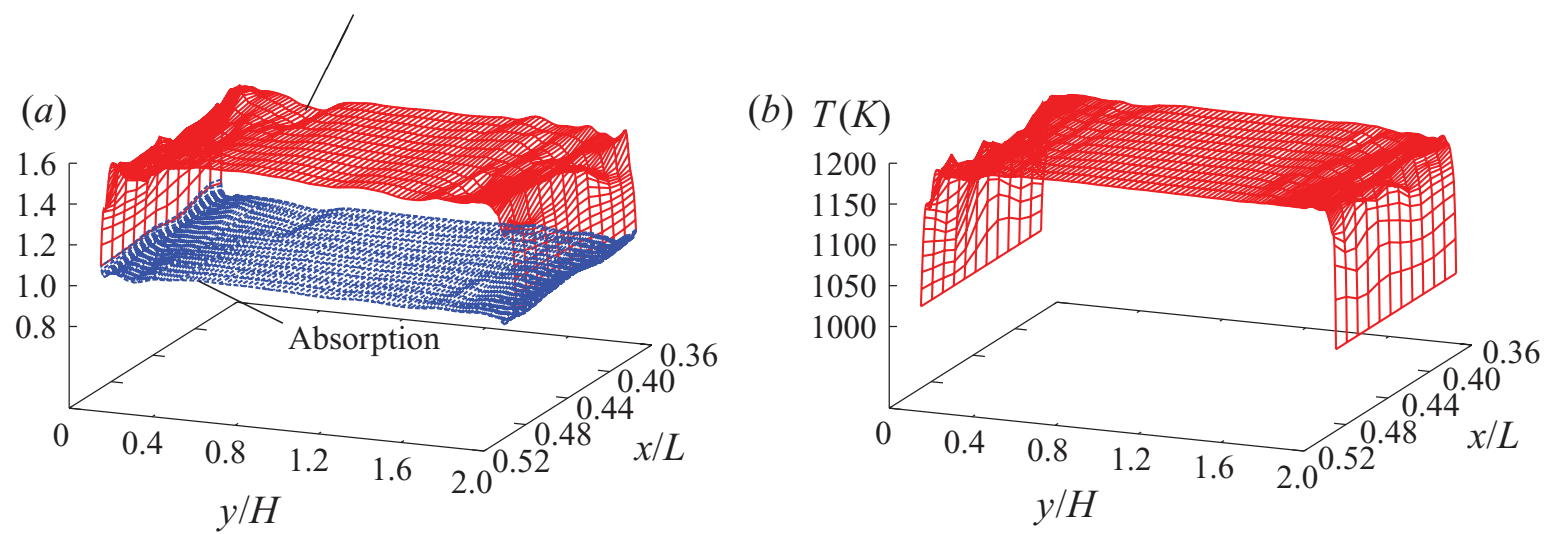

FIGURE 15. (Colour online available at journals.cambridge.org/FLM) (a) Snapshots of emission and absorption integrals (2.8) for Channel 1, normalized with the emission at wall temperature. ( $b$ ) Snapshot of the temperature field used to compute the emission and absorption fields shown in $(a)$.
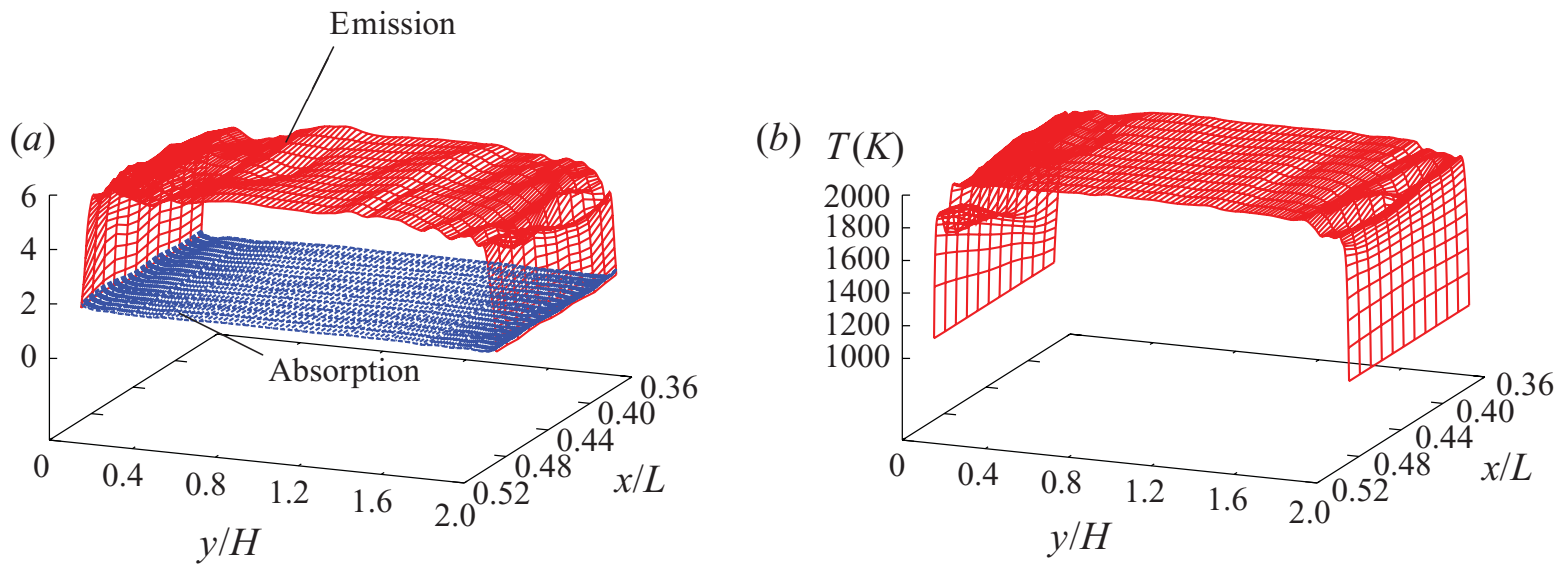

Figure 16. (Colour online) (a) Snapshots of emission and absorption integrals (2.8) for Channel 2, normalized with the emission at wall temperature. (b) Snapshot of the temperature field used to compute the emission and absorption fields shown in $(a)$.

We conclude that, although the source term due to radiation in the energy balance (2.3) increases dramatically with the Mach number, TRI loses importance.

\subsection{Case of a fictitious grey gas with a temperature-dependent Planck mean absorption coefficient}

The previous flow cases have revealed weak effects of radiation on the statistics of the velocity vector and the state variables $\rho, T$, etc., as a result of the very low optical thickness of the gas layer between the channel walls. A straightforward way to increase $\tau_{H}$ would be to enlarge the plate distance $H$. An order of magnitude increase in $H$ would, however, lead to Reynolds numbers which cannot be handled with the presently available supercomputer power. An alternative way is to enhance the absorption coefficient artificially. We adopt this possibility suggested by a referee and assume the following form of the Planck mean absorption coefficient:

$$
\kappa_{P}=C_{k}\left[c_{o}+c_{1}\left(\frac{A}{T}\right)+c_{2}\left(\frac{A}{T}\right)^{2}+c_{3}\left(\frac{A}{T}\right)^{3}+c_{4}\left(\frac{A}{T}\right)^{4}+c_{5}\left(\frac{A}{T}\right)^{5}\right] .
$$

This form was used by Gupta et al. (2009). The coefficients $c_{o}-c_{5}$ and $A$ were taken from a radiation model suggested for water vapour (Sandia National Laboratories 


\begin{tabular}{llllll} 
Flow & \multicolumn{1}{c}{$R e_{\tau}$} & \multicolumn{1}{c}{$M_{\tau}$} & \multicolumn{1}{c}{$R e_{m}$} & \multicolumn{1}{c}{$M_{m}$} & $\tau_{H}$ \\
SNB-cK & $1026(1041)$ & $0.0681(0.068)$ & $16477(17059)$ & $1.26(1.32)$ & 0.006 \\
Grey gas & 1027 & 0.0684 & 16481 & 1.265 & 0.0062
\end{tabular}

TABLE 3. Flow parameters for Channel 1 computed with the SNB-cK model and with the grey gas model, coefficient $C_{k}=0.0121$ (values in brackets correspond to the case without radiation).
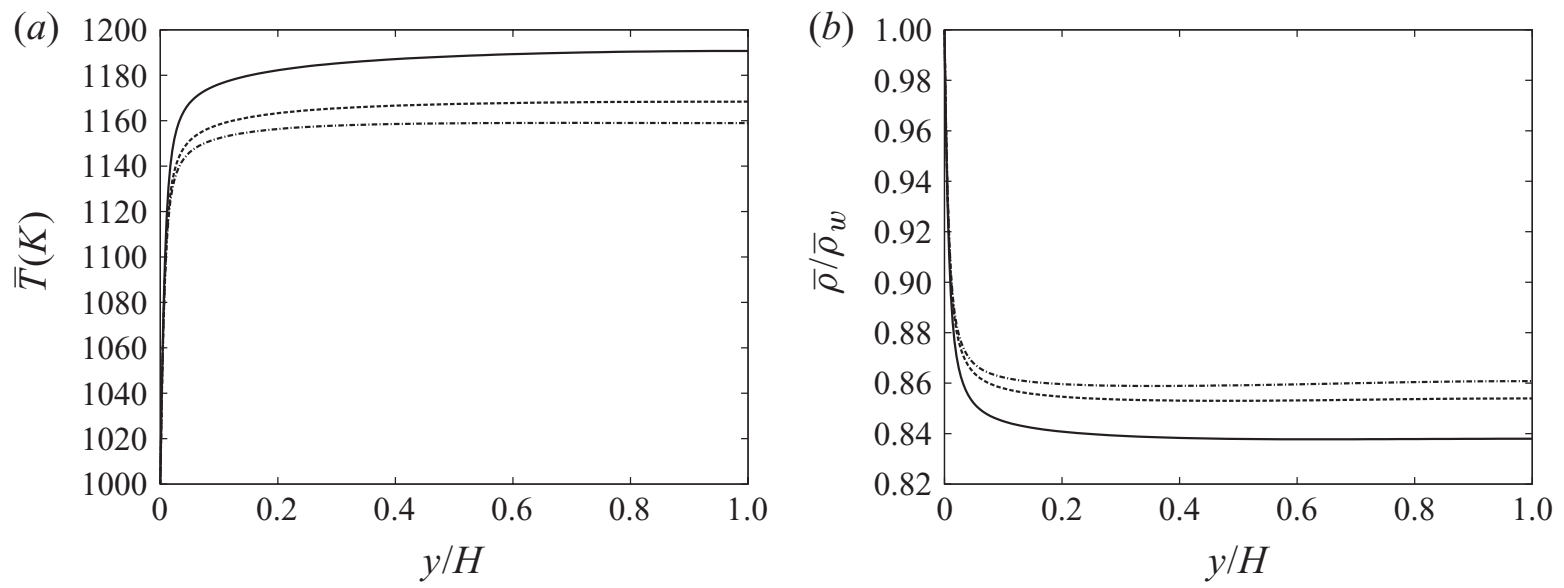

FiguRE 17. Mean temperature $(a)$ and mean density distribution $(b)$. Dashed line: SNB-cK, dash-dotted line: grey gas, solid line: without radiation.

Combustion Research Facility, International Workshop on Measurements and Computation of Turbulent Nonpremixed Flames, 2002). The coefficient $C_{k}$ allows for a variation of the optical thickness independent of the other parameters. In this study we have chosen the values $C_{k}=0.0121,0.1$.

In order to check the behaviour of model equation (4.11), we have recomputed Channel 1 with the above Planck mean absorption coefficient and the specific value $C_{k}=0.0121$. Here, we contrast the results with those of Channel 1, obtained with the SNB-cK model. The comparison is, as before, based on equal friction Reynolds, friction Mach numbers and optical thicknesses. The bulk Reynolds and Mach numbers are, however, quite close for the two radiation models (see table 3 ).

We find very good agreement between the previous velocity statistics and the new ones, but with some difference in the statistics of the temperature and density fields which are partly due to a $3 \%$ higher optical thickness in the grey gas case. It leads to mean temperatures which are below the result for the spectrally resolved absorption coefficient and to mean densities which are above. The r.m.s. temperature/density fluctuations reflect a behaviour which is consistent with that of the mean temperature/density, namely slightly lower/higher values than those obtained with the SNB-cK model (not shown). The three normal Reynolds stresses, the mean velocity and the pressure-strain correlations in the streamwise and spanwise Reynolds stress balances, in figures 18-20, do not noticeably reflect the $3 \%$ difference in the optical thickness. This can be explained by the fact that a radiation source term appears in the energy balance, but not in the momentum balance. Hence, only the temperature and density fields are directly affected by radiation transport. The velocity components depend on radiation only through the density and viscosity variations with temperature (Viskanta 1998). Now, since the flow in the channel 

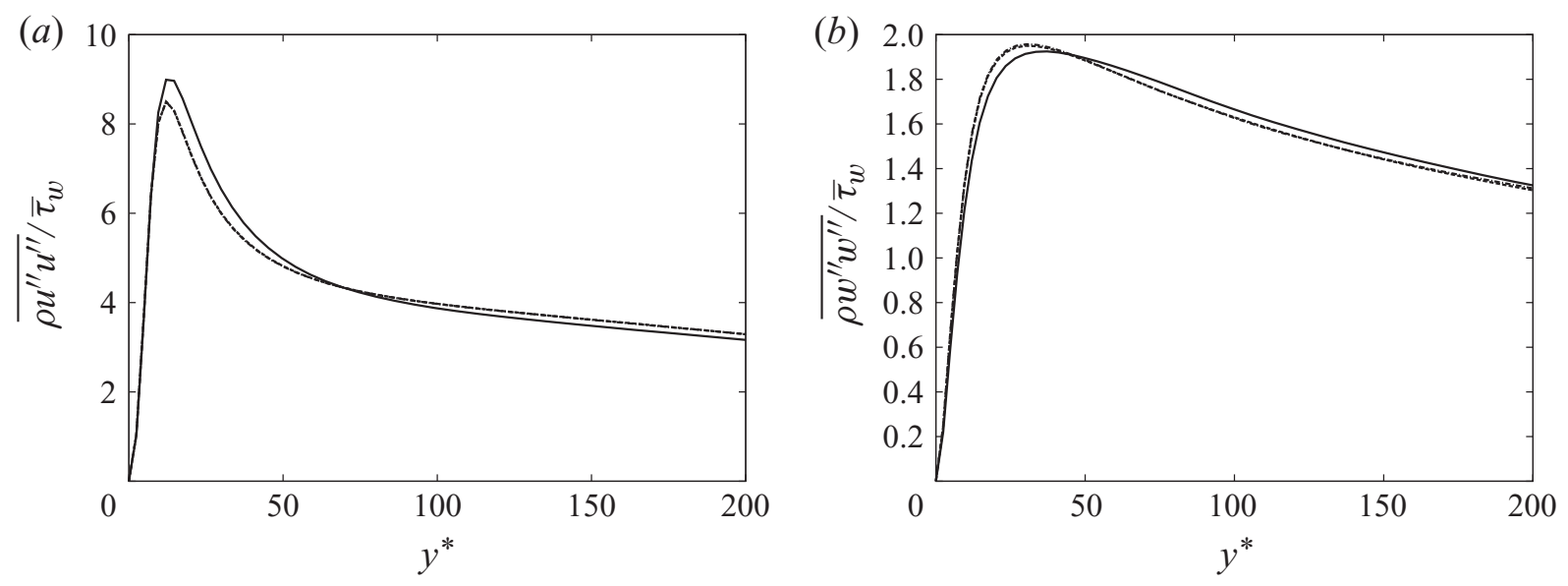

FiguRE 18. Streamwise $(a)$ and spanwise $(b)$ Reynolds stresses. Lines as in figure 17.
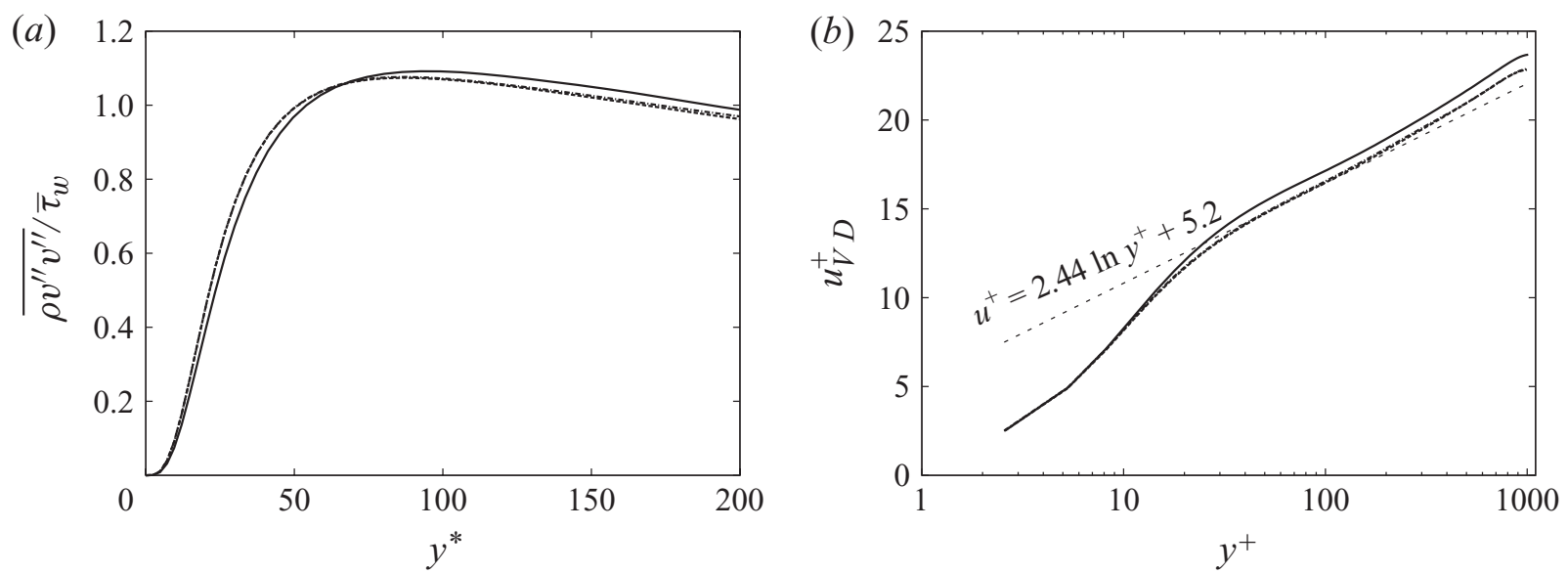

FIGURE 19. Wall-normal Reynolds stress $(a)$ and Van Driest transformed velocity $(b)$. Lines as in figure 17.
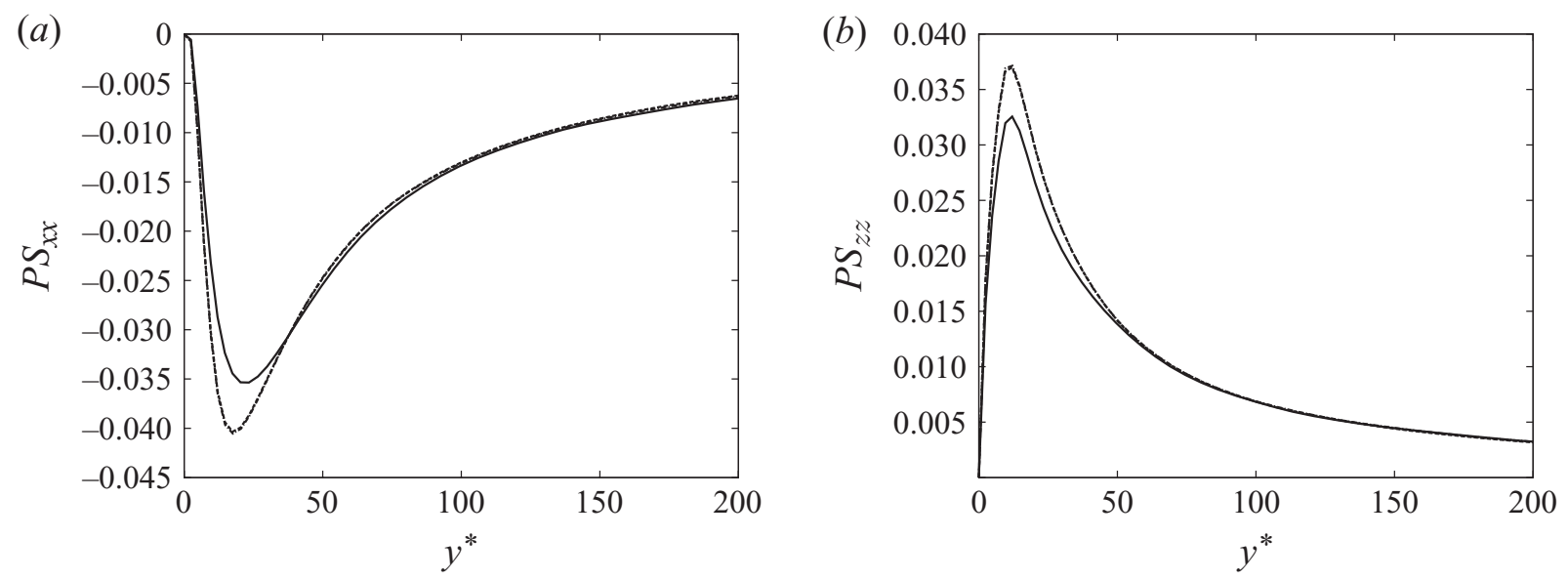

FIGURE 20. Pressure-strain correlations $P S_{x x}(a)$ and $P S_{z z}(b)$ normalized with $\bar{\tau}_{w}^{2} / \bar{\mu}$. Lines as in figure 17.

core is fully turbulent and independent of viscosity for the present friction Reynolds numbers, effects of radiation should be more pronounced in the wall layer, where viscosity still plays a role. This is clearly seen in figures 18 and 20 . The pressurestrain correlation profiles (figure 20) lie on top of each other for the two radiation models. The above results hence document the fact that a simple grey gas model and the spectrally resolved narrow-band model practically lead to the same velocity 


$\begin{array}{lccccc}\text { Flow } & R e_{\tau} & M_{\tau} & R e_{m} & M_{m} & \tau_{H} \\ \text { Radiation turned off } & 982 & 0.07 & 16700 & 1.29 & - \\ \text { Grey gas } & 977 & 0.071 & 16620 & 1.28 & 0.04\end{array}$

TABLE 4. Flow parameters for Channel 1 without radiation and with the artificially increased absorption coefficient according to the grey gas model (4.11) (coefficient $C_{k}=0.1$ ).
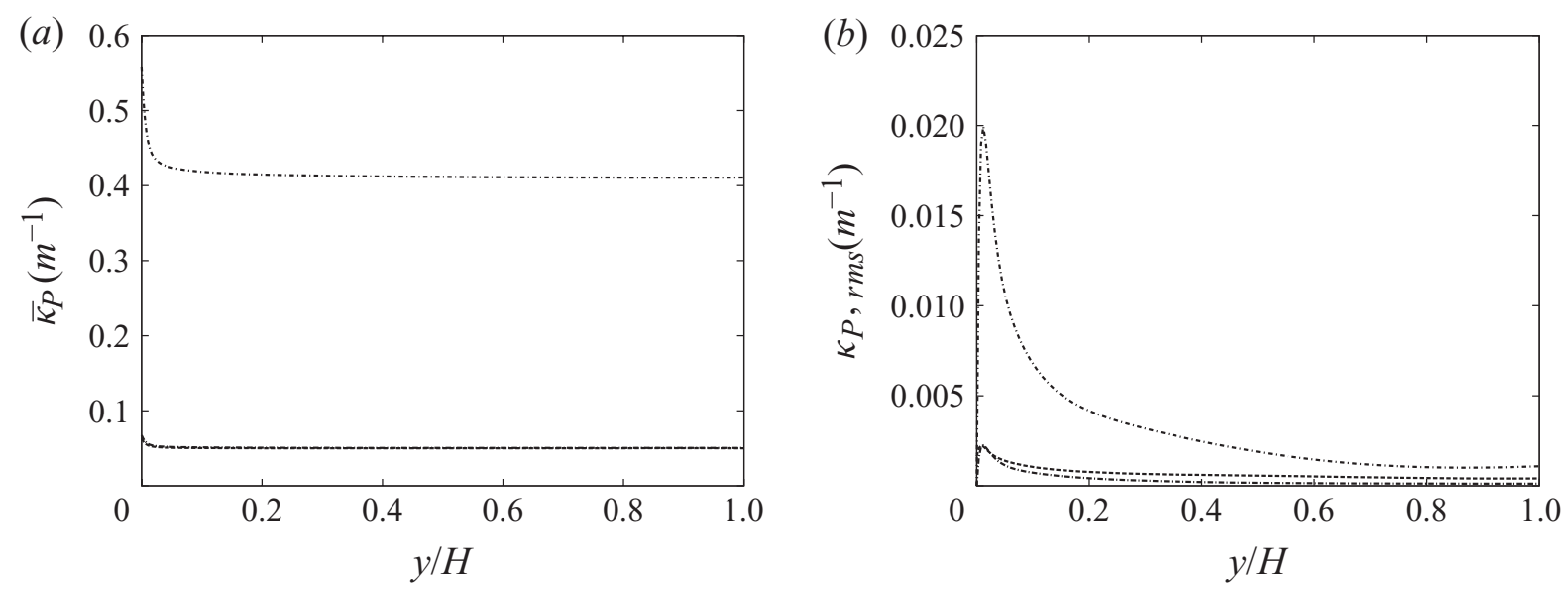

FIGURE 21. Space-time averaged Planck mean absorption coefficient $(a)$ and its r.m.s. fluctuations $(b)$ according to the SNB-ck model (dashed line) and the grey gas model (dash-dotted lines).

and pressure statistics in the present case of an optically very thin gas layer. We complement this comparison with plots of the space-time averaged Planck mean absorption coefficient (figure 21a) and its r.m.s. value (figure 21b) for both models across the channel, as obtained from the resolved fluctuating temperature fields. The figures also contain the profiles for the case of a grey gas with an artificially increased absorption coefficient $\left(C_{k}=0.1\right)$. This case reveals a slight increase of $\bar{\kappa}_{P}$ from the channel centreline towards the wall and a steep increase close to the wall, where the temperature rapidly falls to its wall value of $1000 \mathrm{~K}$. The steep increase in $\bar{\kappa}_{P}$ produces peak values in its r.m.s. fluctuations close to the wall.

We now discuss the case of an increased optical thickness under the assumption of a grey gas and its comparison with the case where radiation is turned off. In contrast to the previous computations in this section and the discussion in $\S 4.2$, we adjust the mass flux when radiation is turned off, so that it matches with that under the effect of radiation. The flow parameters in both cases are specified in table 4. Although the optical thickness is still fairly low $(4 \%)$, the effect of radiation on the state variables can now be specified as very noticeable. From figure 22 we conclude that the temperature increase in the channel core (and in turn the density decrease) due to energy dissipation at supersonic speeds is to a good deal removed through radiative energy transfer towards the wall $(y=0)$. The modified shape of the mean temperature and density profiles has to be seen in the light of the changes observed in the heat balance (4.7), the terms of which are displayed in figure 23. We note that the radiative heat flux is now comparable to the conductive heat flux close to the wall. For $y^{*} \leqslant 21$, both fluxes are directed towards the wall. At $y^{*}=21$, the conductive heat flux changes sign and now counteracts the radiative flux which always points towards the wall. A similar behaviour is also characteristic of the turbulent heat flux which transports 

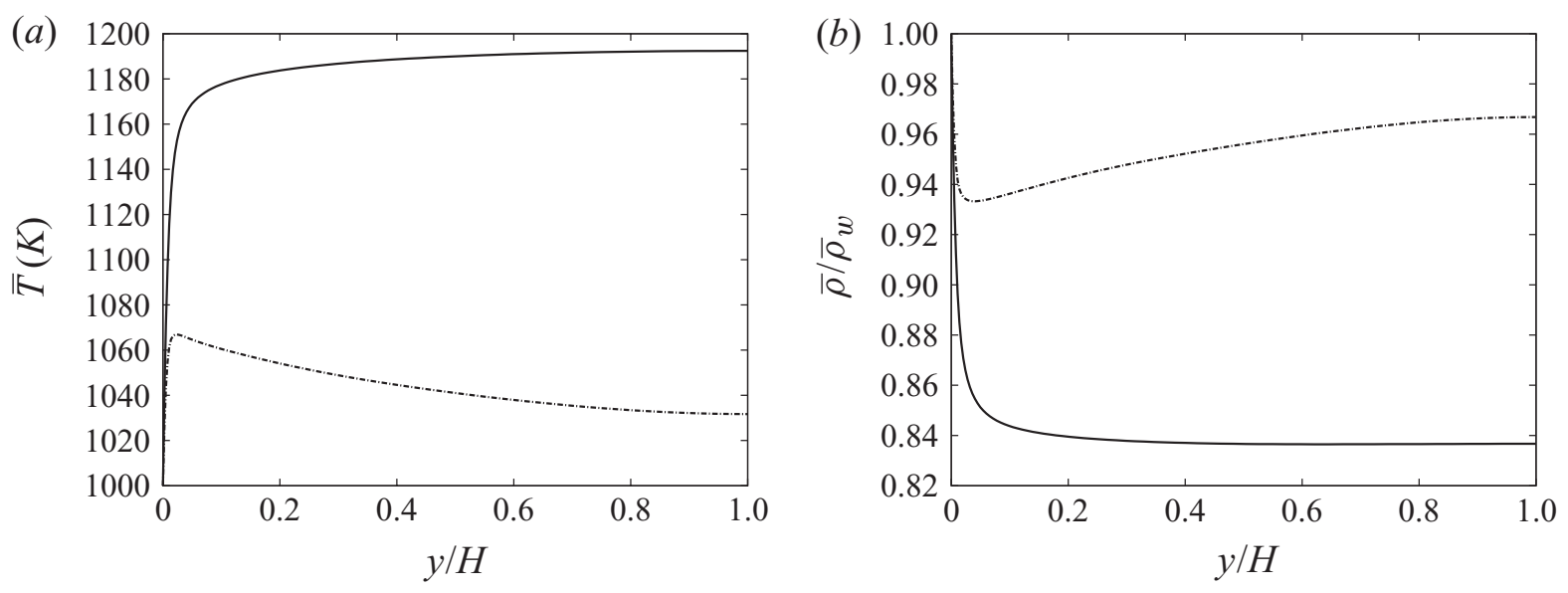

Figure 22. Mean temperature $(a)$ and mean density distribution $(b)$ : Dash-dotted line: grey gas, $\tau_{H}=0.04$, solid line: without radiation.
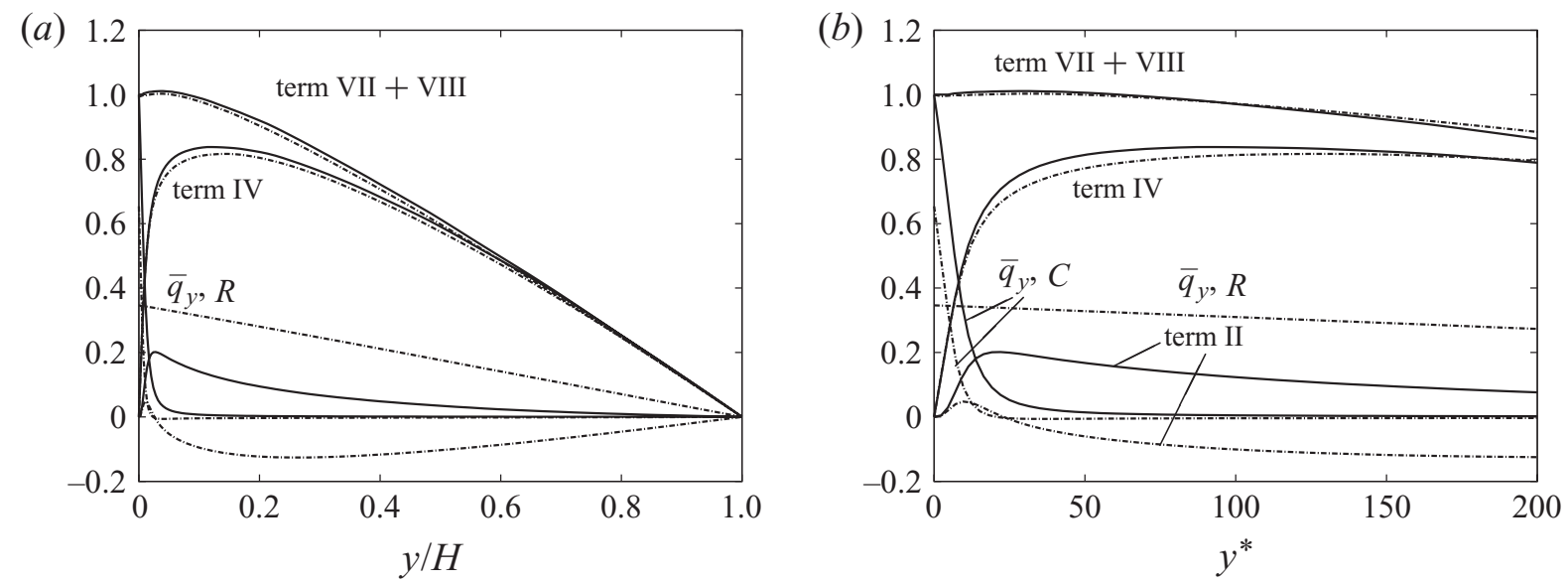

FIgURE 23. Heat flux scaled by the modulus of the total heat flux at the wall. Lines as in figure 22.
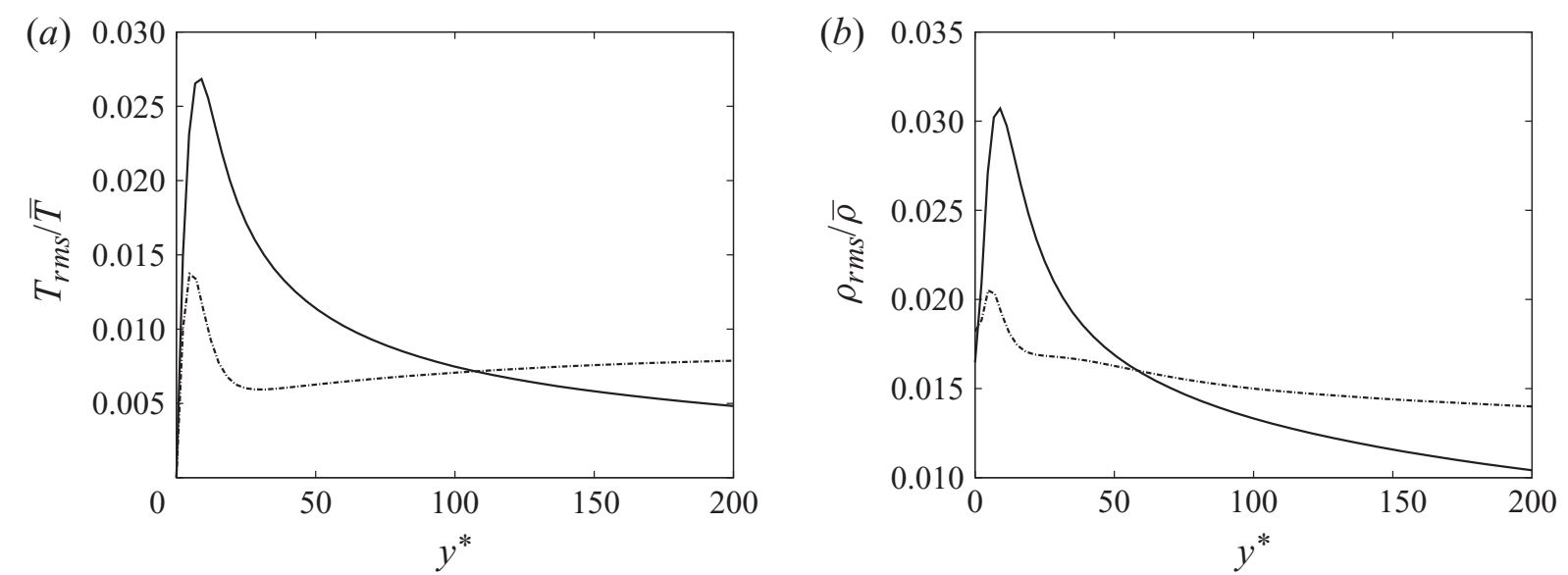

FigURE 24. RMS temperature $(a)$ and density $(b)$ fluctuations. Lines as in figure 22.

energy towards the wall in a thin near-wall layer, $0 \leqslant y^{*} \leqslant 23$, but beyond $y^{*} \approx 23$ it points towards the channel core and stays negative until close to the symmetry plane as a result of the slope of the mean temperature profile. Effects, similar in strength to those of the mean temperature and density, are also observed in the temperature and density fluctuations, see figure 24 . We observe reductions by factors of nearly 2 in the wall layer and enhancements of comparable order of magnitude away from 

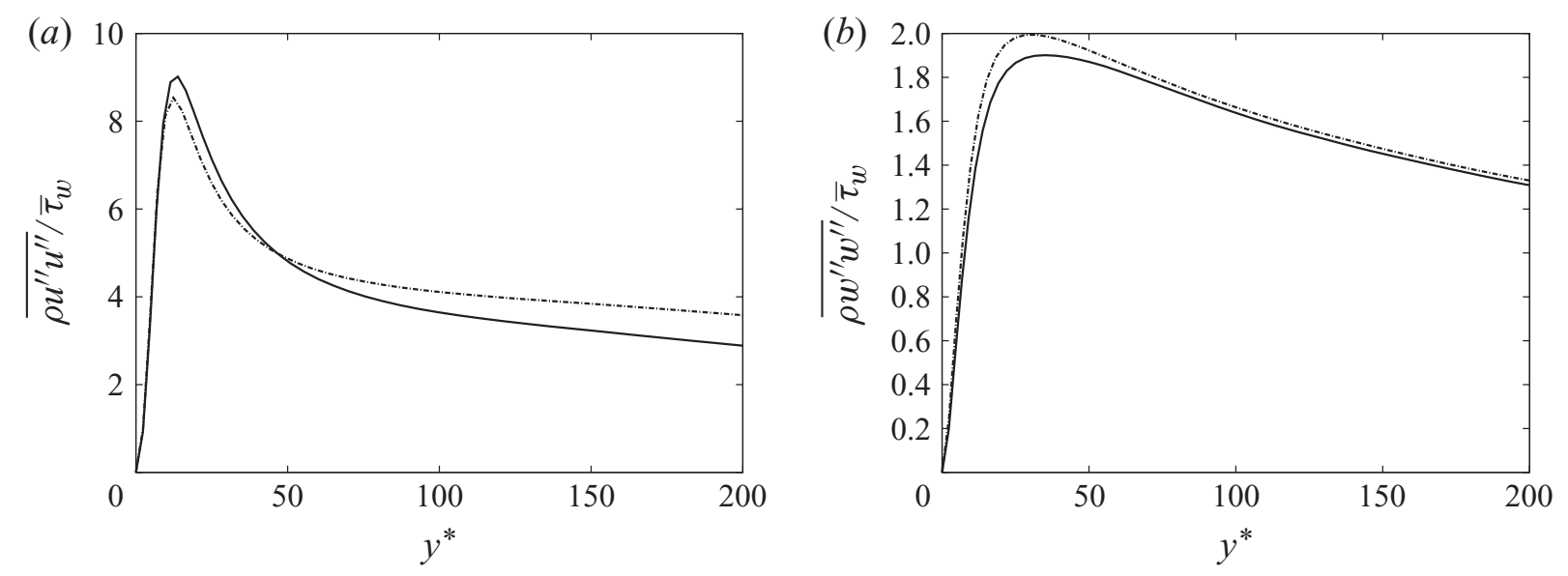

FiguRE 25. Streamwise $(a)$ and spanwise $(b)$ Reynolds stresses. Lines as in figure 22.
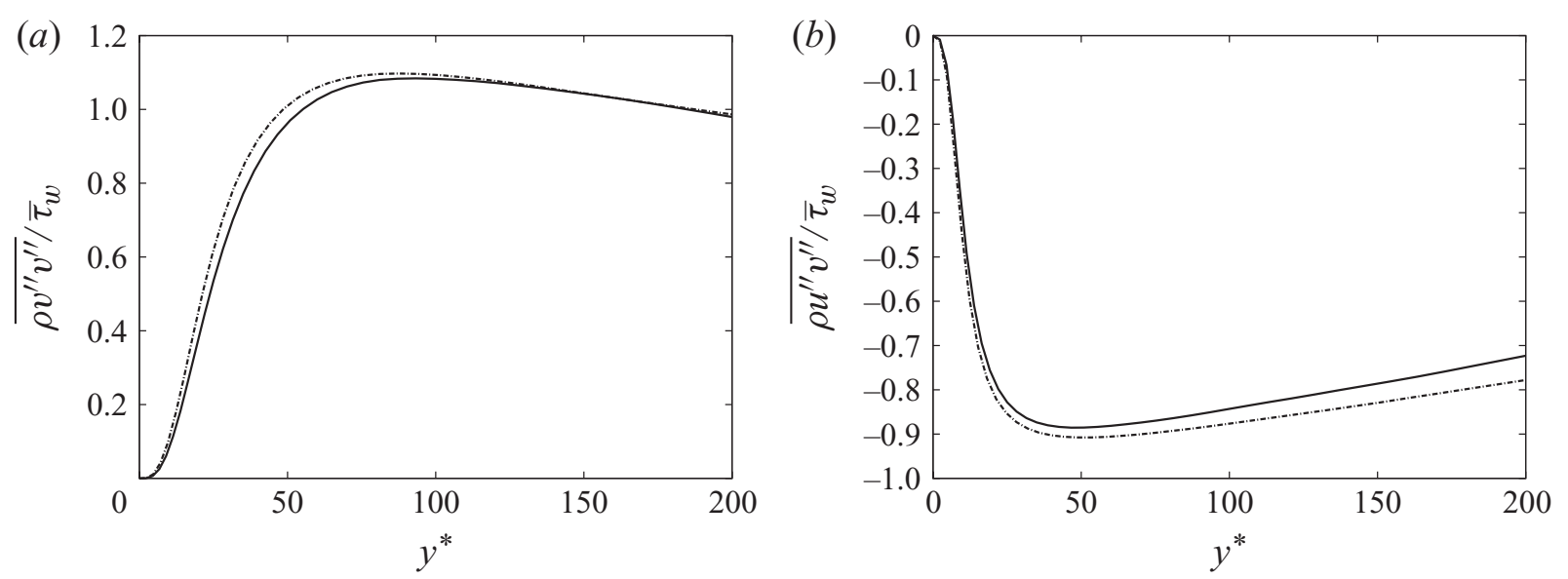

FiguRE 26. Wall-normal Reynolds stress $(a)$ and Reynolds shear stress $(b)$. Lines as in figure 22 .

the wall. It remains to discuss the effect of radiation on the Reynolds stresses and the pressure-strain correlations. Figures 25 and 26 show all four components of the Reynolds stresses. When we compare the present effect of radiation with that at the lower Planck mean absorption coefficient and lower optical thickness (figures 6 and 7), we note that the previous trends are confirmed, i.e. we observe a decrease in the streamwise stress and an increase in all remaining stresses in the wall layer. The effect that radiation counteracts compressibility is even slightly enhanced in this range. Similar trends hold for the r.m.s. velocity fluctuations, normalized with the friction velocity (not shown here). We also present the streamwise and wall-normal Reynolds stresses for the complete wall-normal half-domain in figure 27 to show that the behaviour seen around $y^{*}=200$ persists in the channel core. A similar behaviour is found for the remaining components. Radiation clearly counteracts the Mach-number (compressibility) effect in the channel core via changes of the mean temperature and density fields. The pressure-strain correlations in figure 28 , normalized by the ratio of wall-shear stress squared and the local mean viscosity, show larger deviations, in the near-wall region, from those where radiation is turned-off, than in the case of very low absorption coefficient (figure 8). Thus, an increase in the absorption coefficient enhances the effect of radiation on the pressure-strain correlations and confirms the previously found tendencies. Last not least, the shift of the mean Van Driest transformed velocity towards the logarithmic law in the log region, which is presented in figure $3(b)$, is confirmed in figure 29 . 

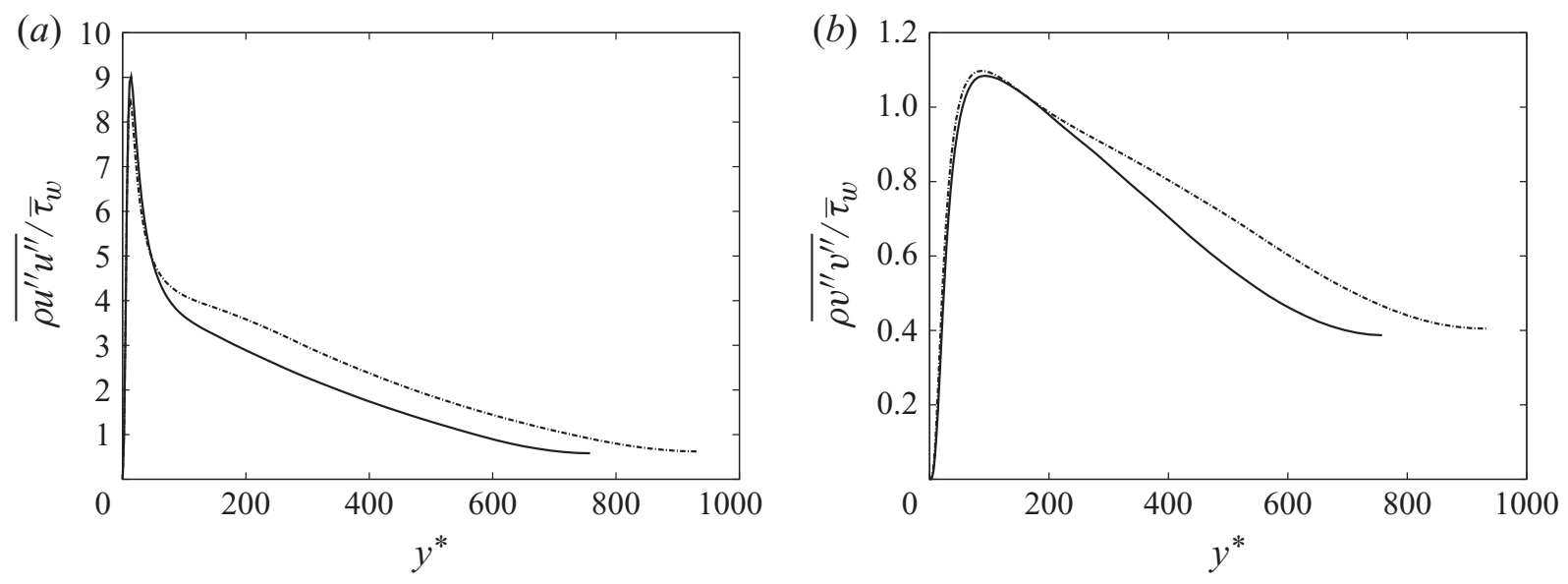

FiguRE 27. Streamwise $(a)$ and wall-normal $(b)$ Reynolds stresses. Lines as in figure 22.
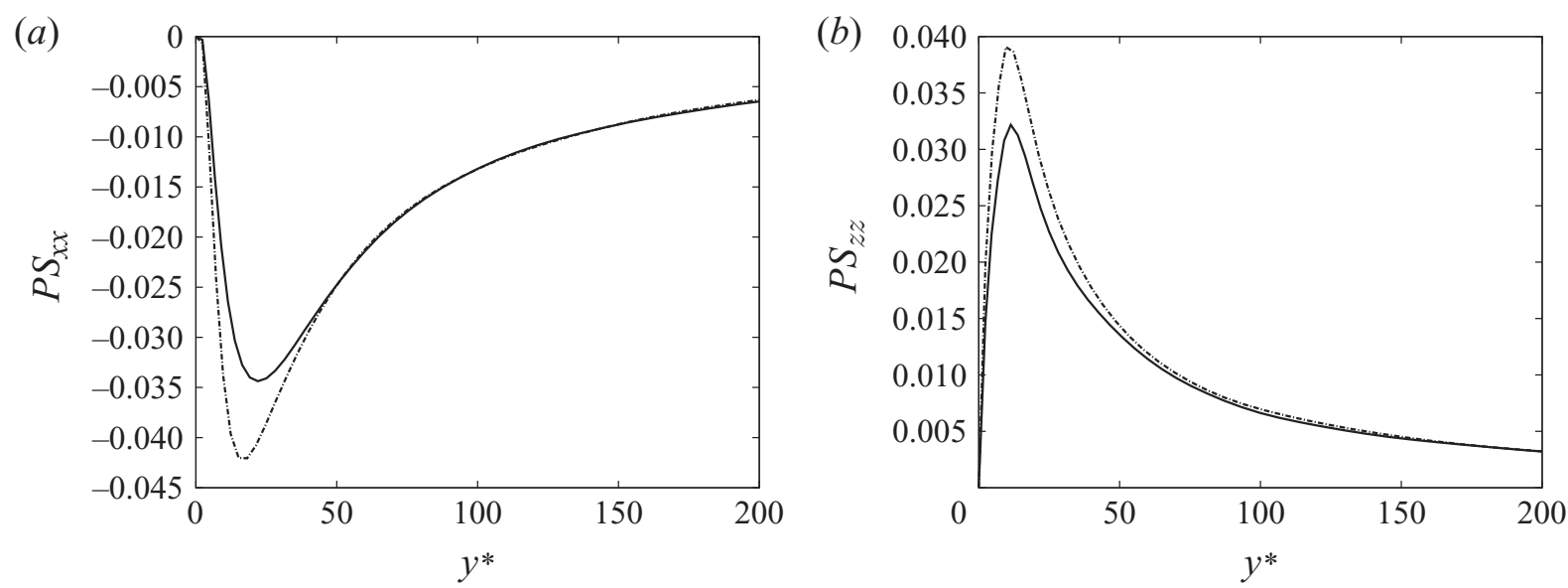

FIGURE 28. Pressure-strain correlations $P S_{x x}(a)$ and $P S_{z z}(b)$ normalized with $\bar{\tau}_{w}^{2} / \bar{\mu}$. Lines as in figure 22.

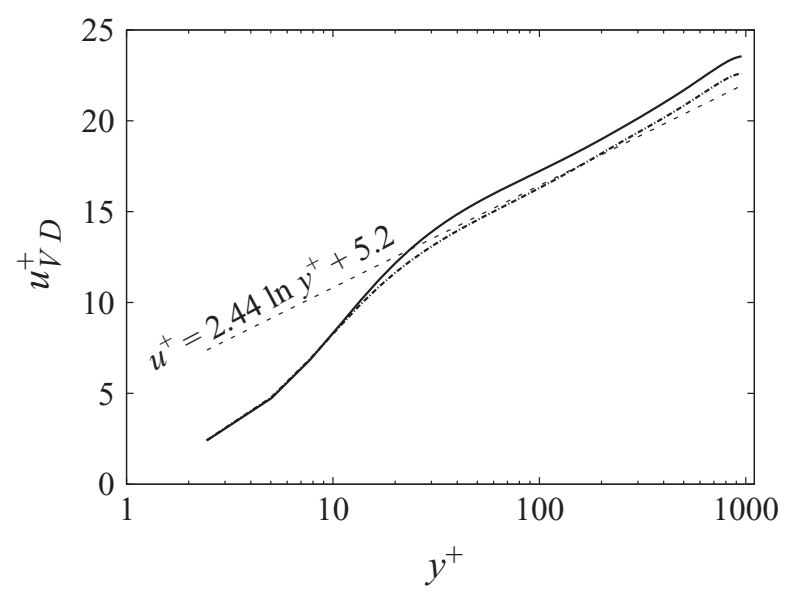

Figure 29. Van Driest transformed mean streamwise velocity. Lines as in figure 22.

\section{Concluding remarks}

Radiative heat transfer plays an important role in combustion processes, but it also produces noticeable effects in non-reacting flows, provided the optical thickness through which the photons travel has some non-zero value. The present work clearly shows, through fully coupled simulations, in which way radiation modifies 
the structure of supersonic turbulent channel flow, when there are no reactions and the friction Reynolds numbers are of the order of 1000. We have chosen water vapour as working fluid since it is one of the important combustion gases and have accounted for its radiative properties using the expensive SNB-cK model. LESs have been performed with high-order numerical methods in a minimal channel at two bulk Mach numbers of 1.26 and 2.88. The corresponding optical thicknesses based on the mean Planck absorption coefficient are 0.006 and 0.003 , i.e. very low, indicating weak interaction between turbulence and radiation.

Both flow cases, nevertheless, demonstrate that effects of radiation counteract effects of compressibility. While non-radiating fully developed supersonic channel flow shows an increase in the normalized streamwise Reynolds stress and reductions in the other stress components compared to incompressible flow, thermal radiation dampens the streamwise stress and enhances the other stresses in the wall layer $\left(0 \leqslant y^{*} \leqslant 50\right)$. This behaviour is a result of the decrease (Mach-number effect) or increase (radiation effect) of the corresponding pressure-strain correlations in the wall layer. The mean heat flux balance reflects a decrease in the mean heat flux by conduction, in the turbulent heat flux and in the work done by the viscous and Reynolds stresses due to radiation. The radiative heat flux, which grows linearly from a zero value in the symmetry plane, reaches a value of only $4 \%$ of the total heat flux at the wall (Channel 1). This means that the radiative heat flux at the wall might have been computed from the flow data without performing expensive simulations where the turbulent and radiative fields are fully coupled at every convective time interval.

Computation of the instantaneous emission and absorption integrals reveals that although both integrals increase with the Mach number, leading to higher radiative heat fluxes, TRI becomes weaker. This is partly due to the fact that the absorption coefficient decreases at higher temperature levels and partly because the channel width (and with it the mean optical thickness) has to be reduced when the mass flux goes up (from Channels 1 to 2 ), in order to keep the Reynolds numbers roughly fixed.

To reach a higher optical thickness, we have assumed the grey gas model (4.11) and have increased the coefficient $C_{k}$. That way the optical thickness goes up to $4 \%$ keeping the channel half-width unchanged and the Reynolds number remains in a range accessible to wall-resolved LES. An LES without radiation at the same bulk Reynolds and Mach numbers has also been performed for comparison.

It turns out that an optical thickness of $4 \%$ reduces the mean temperature increase due to dissipation by a factor of roughly 6 . The heat flux by radiation is now comparable to that by conduction in the wall layer. The effect of radiation on the second-order turbulence statistics, which was found at very low optical thickness of 0.006 , is now confirmed in its tendencies and quantitatively enhanced.

These findings allow for the conclusion that radiation affects the turbulence structure in supersonic shear layers in a non-negligible way, when the shear layer has a high enough optical thickness.

The first author (S.G.) thanks the Deutsche Forschungsgemeinschaft for financial support in the framework of the project FR 478/24-1. The authors are grateful to Prof. Joseph Mathew of IISc, Bangalore, for valuable discussions and suggestions on the first draft of this paper. They also thank the reviewers for their comments and suggestions. 


\section{REFERENCES}

Adams, N. A. \& LeONARD, A. 1999 Deconvolution of subgrid scales for the simulation of shockturbulence interaction. In Direct and Large-Eddy Simulation III (ed. P. Voke, N. D. Sandham \& L. Kleiser), p. 201, Kluwer Academic Publishers, Dordrecht.

Amaya, J., Cabrit, O., Poitou, D., Cuenot, B. \& Hafi, M. El 2010 Unsteady coupling of NavierStokes and radiative heat transfer solvers applied to an anisothermal multi-component turbulent channel flow. J. Quant. Spectrosc. Radiat. Transfer 111, 295-301.

Coelho, P. J. 2007 Numerical simulation of the interaction between turbulence and radiation in reactive flows. Prog. Energy Combustion Sci. 33, 311-383.

Coelho, P. J. 2009 Approximate solutions of the filtered radiative transfer equation in large-eddy simulations of turbulent reactive flows. Combust. Flame 156, 1099-1110.

Coleman, G. N., Kim, J. \& Moser, R. D. 1995 A numerical study of turbulent supersonic isothermal-wall channel flow. J. Fluid Mech. 305, 159-183.

ERN, A. \& Giovangigli, V. 1995 Fast and accurate multicomponent transport property evaluation. J. Comput. Phys. 120, 105-116.

Foysi, H., Sarkar, S. \& Friedrich, R. 2004 Compressibility effects and turbulence scalings in supersonic channel flow. J. Fluid Mech. 509, 207-216.

Gardiner, W. 1984 Combustion Chemistry. Springer.

Ghosh, S., Sesterhenn, J. \& Friedrich, R. 2006 DNS and LES of compressible turbulent pipe flow with isothermal wall. In Direct and Large Eddy Simulation VI(ed. E. Lamballais, R. Friedrich, B. J. Guerts \& O. Métais) pp. 721-728. Springer.

Ghosh, S., Sesterhenn, J. \& Friedrich, R. 2008 Large-eddy simulation of supersonic turbulent flow in axisymmetric nozzles and diffusers. Intl J. Heat Fluid flow pp. 579-590.

Gupta, A., Modest, M. F. \& Haworth, D. C. 2009 Large-eddy simulation of turbulence-radiation interactions in a turbulent planar channel flow. J. Heat Transfer 131, 061704.

Huang, P. G., Coleman, G. N. \& Bradshaw, P. 1995 Compressible turbulent channel flows: DNS results and modelling. J. Fluid Mech. 305, 185-218.

Jensen, K., Ripoll, J-F., Wray, A., Joseph, D. \& Hafi, M. El 2007 On various modelling approaches to radiative heat transfer in pool fires. Combust. Flame 148, 263-279.

Jimenez, J. \& Moin, P. 1991 The minimal flow unit in near-wall turbulence. J. Fluid Mech. 225, $213-240$.

Joseph, D., Hafi, M. El, Fournier, R. \& Cuenot, B. 2005 Comparison of three spatial differencing schemes in discrete ordinates method using three-dimensional unstructured grids. Intl J. Therm. Sci. 44, 851-864.

Lechner, R., Sesterhenn, J. \& Friedrich, R. 2001 Turbulent supersonic channel flow. J. Turbul. 2, 1-25.

Lele, S. K. 1992 Compact finite difference schemes with spectral-like resolution. J. Comput. Phys. $103,16-42$.

Liu, F., Smallwood, G. J. \& Gulder, O. L. 2000 Application of the statistical narrow-band correlated-k model to low resolution spectral intensity and radiative heat transfer calculationseffects of the quadrature scheme. Intl J. Heat Mass Transfer 43, 3119-3135.

Mathew, J., Lechner, R., Foysi, H., Sesterhenn, J. \& Friedrich, R. 2003 An explicit filtering method for large eddy simulation of compressible flows. Phys. Fluids 15, 2279-2289.

Modest, M. F. 2003 Radiative Heat Transfer. 2nd edn. Academic Press.

Poitou, D., Amaya, J., Chandra, B. S., David, J., Hafi, M. El \& Cuenot, B. 2009 Validity limits for the global model FS-SNBcK for combustion applications. In Proceedings of Eurotherm83 - Computational Thermal Radiation in Participating Media III, Lisbon, Portugal.

SesterhenN, J. 2001 A characteristic-type formulation of the Navier-Stokes equations for high order upwind schemes. Comput. Fluids 30, 37-67.

SoUfiani, A. \& TAINE, J. 1997 High temperature gas radiative property parameters of statistical narrow-band model for $\mathrm{H}_{2} \mathrm{O}, \mathrm{CO}_{2}$ and $\mathrm{CO}$, and correlated-k model for $\mathrm{H}_{2} \mathrm{O}$ and $\mathrm{CO}_{2}$. Intl J. Heat Mass Transfer 40, 987-991.

Stolz, S. 2000 Large eddy simulation of complex shear flows using an approximate deconvolution model. PhD thesis, ETH, Zurich.

Stolz, S. \& AdAms, N. A. 1999 An approximate deconvolution procedure for large-eddy simulation. Phys. Fluids 11, 1699-1701. 
Stolz, S., Adams, N. A. \& Kleiser, L. 2001 An approximate deconvolution model for large-eddy simulation with application to incompressible flows. Phys. Fluids 13, 997-1015.

Viskanta, R. 1998 Overview of convection and radiation in high temperature gas flows. Intl J. Engng Sci. 36, 1677-1699.

Williamson, J. K. 1980 Low-storage Runge-Kutta schemes. J. Comput. Phys. 35, 48-56.

Wu, Y., Haworth, D. C., Modest, M. F. \& Cuenot, B. 2005 Direct numerical simulation of turbulence/radiation interaction in premixed combustion systems. Proc. Combust. Inst. 30, 259-266. 